Math. Model. Nat. Phenom.

Vol. 2, No. 4, 2007, pp. 74-104

\title{
Pattern Formation of Competing Microorganisms in Sediments
}

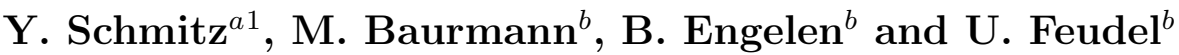 \\ ${ }^{a}$ Systems Biology and Bioinformatics Group, Department of Computer Science, \\ University of Rostock, Germany \\ ${ }^{b}$ Institute of Chemistry and Biology of the Marine Environment, \\ University of Oldenburg, Germany
}

\begin{abstract}
We present a three species model describing the degradation of substrate by two competing populations of microorganisms in a marine sediment. Considering diffusion to be the main transport process, we obtain a reaction diffusion system (RDS) which we study in terms of spontaneous pattern formation. We find that the conditions for patterns to evolve are likely to be fulfilled in the sediment. Additionally, we present simulations that are consistent with experimental data from the literature. We focus on the interaction between pattern formation and non-uniform forcing, which naturally occurs in our model, since layers closer to the sea water are richer in particular chemicals than deeper layers. We find that heterogeneous forcing has a strong impact on the structures that evolve, leading to the formation of banded structures or even pulses instead of the well-known Turing patterns.
\end{abstract}

Key words: Turing instability, pattern formation, population dynamics, competition AMS subject classification: 70K50, 37G35, 92D25, 92D40, 34C60

\section{Introduction}

Since A.M.Turing [49] published his seminal paper on morphogenesis in 1952, there has been a vast literature on both the theoretical and practical aspects of spontaneous pattern formation in reaction diffusion systems (RDS) (see, for example, [32, 13, 41, 37]). Turing models have been applied to such diverse areas as biology and chemistry [26], ecology [44, 31, 4, 1],

\footnotetext{
${ }^{1}$ Corresponding author. E-mail: schmitz@informatik.uni-rostock.de
} 
semiconductor physics [2], material sciences [51], hydrodynamics [52], astrophysics [34] and even economics [28].

Turing formulated two essential conditions for spontaneous pattern formation to take place in RDS: On the one hand the growth of one reactant has to be autocatalytic (a presupposition that exists in a weaker formulation for systems with more than two reactants), on the other hand there has to be a significant difference in the diffusion coefficients of the reactants. The patterns, which evolve, are characterized by a spatially inhomogeneous distribution of the chemical reactants.

Since it is difficult to meet both conditions, attempts to obtain experimental evidence of Turing structures remained unsuccessful for nearly four decades. The first experimental evidence of stationary Turing structures was presented by Castets et al. [14]. They used an open reactor system, wherein reactants were continuously fed into the system to keep it far from equilibrium. The resulting sustained standing nonequilibrium chemical patterns exhibit all the properties of Turing structures.

Numerical simulations of many autocatalytic model systems, such as the Brusselator model or the reduced Oregonator model of the Belousov-Zhabotinsky reaction, predict that Turing structures can only be generated when diffusion coefficients differ in at least one order of magnitude [40,33]. In chemical experiments, the diffusion coefficients of the reactants, however, show in general much less variation.

The spontaneous formation of spatial patterns cannot only be observed in various fields of biology [32], but also in many ecological systems. Examples from terrestrial ecosystem research are the formation of vegetation patterns in semiarid areas $[23,19,30,55]$ or the spatial patterns of phytoplankton distributions in marine ecosystems [29]. In the mentioned examples, the modelling approach of RDS has been exploited to explain the pattern formation process.

Spatially inhomogeneous distributions of microorganisms like bacteria have also been observed in marine sediments $[9,25]$. These patterns involve fronts and localized spots of high bacterial activity. The emergence of such patterns can be on the one hand due to forcing such as inhomogeneous distributions of substrate for uptake as well as of electron acceptors for respiration of the microorganisms. On the other hand, spontaneous pattern formation due to Turing's mechanism is possible $[5,6]$.

In this paper, we study the degradation of chemical substances in marine sediments that is processed by a complex network of different, mostly microbially catalysed, reactions (see, e.g. [16, 22, 43, 50]). Within this network, different microorganisms and hence different degradation pathways compete with each other. As a representative for the different forms of competition, we consider two species of bacteria competing for one chemical substrate. This three-variable system advantages spontaneous pattern formation: It has been shown that planktonic bacteria can stimulate the growth of cells by excreting signal molecules [11, 12], a process that induces autocatalytic growth in the model. Additionally, the mobility of bacteria and chemicals in the sediment can differ to a high degree. This is due to the fact that bacteria tend to stick in biofilms or to the sediment matrix whereas chemicals move more or less freely in pore space. This property guarantees that we fulfil the necessary relation 
between diffusion coefficients to obtain Turing structures without further ado. Therefore, the marine sediment should be an ideal system to observe spontaneous pattern formation. And indeed, Thar and Kühl [47] have reported complex spatial patterns formed by marine microaerophilic bacteria kept in enrichment cultures under quasi-natural conditions. The sulfide-oxidising bacterium Thiovulum majus, for instance, forms mucus veils on top of sulfidic marine sediment exhibiting regular bacterial patterns in space. These experimentally observed patterns are of exactly the same type, as those that evolve in RDS models.

However, our aim is not only to show that the degradation model is capable of pattern formation, but rather to study the interaction of pattern formation, competition and nonuniform forcing. The latter factor comes into play because the supply of chemicals in natural environments is not homogeneous with respect to depth.

The paper is structured as follows. In the next section we introduce the model. We describe the relevant processes and discuss their biogeochemical background. A list of realistic parameters and the transformation of the model system into a dimensionless form complete the section. Section 3 is concerned with the mathematical background of pattern formation in 3-component reaction diffusion systems. It mainly refers to findings of other authors (namely [41] and [39]), but is placed here to clarify the notation and to specify the conditions for pattern formation in our 3-component system, explicitly. In Section 4, after discussing the properties of the local model, the theoretical findings of section 3 are applied to the spatially extended degradation model to derive the parameter ranges, in which Turing instabilities occur. Numerical simulations showing examples for the interplay of competition, non-uniform forcing and pattern formation are presented in Section 5. We finish this article with a discussion and an outlook (Section 6).

\section{Model formulation}

Our conceptual model describes the degradation of a substrate, namely dissolved organic carbon (DOC) in sediments by two competing populations of microorganisms. The model formally corresponds to a predator-prey-system of two bacterial species (predators) competing for one substrate (prey). The modeled consumption (predation) consists of a microbial catalysed redox reaction. While the substrate DOC, the electron donor, is elementary for the two bacteria species, both species are dependent on different electron acceptors $(E A)$, such as nitrate, sulfate, manganese- and iron-oxides, used for respiration. We focus on mixed tidal flats, where bioirrigation and diffusion are the dominant transport processes [18]. Advection is neglected here, since it is only particularly important in permeable, sandy sediments [20], which are not considered in this paper. Figure 1 shows the general structure of our model.

The model is based on a benthic microorganism-substrate system proposed by [6]. It has been modified to incorporate the competition of the predators. Our three components - substrate (Dissolved Organic Carbon) $U$ and two different bacteria populations $V$ and $W$ - are assumed to be homogeneously distributed, when transport processes are neglected. Diffusion within the sediment is isotropic. Moreover, we suppose that the sea water above the sediment is a reservoir with a constant high substrate concentration $U_{0}$. 

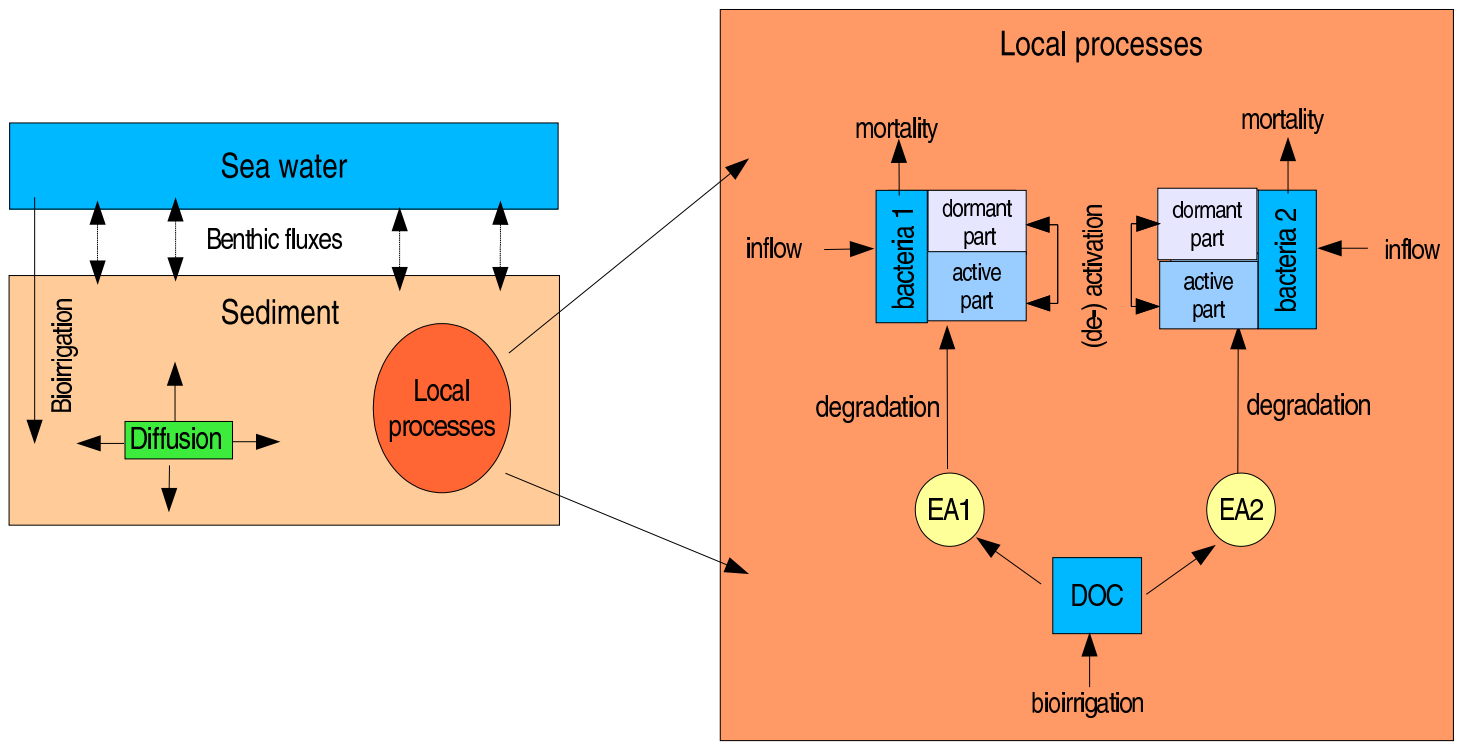

Figure 1: Model of competing microorganisms in the sediment

The dynamics of the 3-component system is represented by three coupled partial differential equations:

$$
\begin{aligned}
\frac{\partial U}{\partial \tau} & =\sigma\left(U_{0}-U\right)-\beta_{V} F_{V}(U)-\beta_{W} F_{W}(U)+D_{U} \nabla^{2} U \\
& =\text { bioirrigation - consumption by } \mathrm{V}-\text { consumption by } \mathrm{W}+\text { diffusion } \\
\frac{\partial V}{\partial \tau} & =\alpha_{V} F_{V}(U) \frac{A}{H_{A}+A}-M_{V} V+E_{V}+D_{V} \nabla^{2} V \\
& =\text { growth }- \text { mortality }+ \text { inflow }+ \text { diffusion } \\
\frac{\partial W}{\partial \tau} & =\alpha_{W} F_{W}(U) \frac{B}{H_{B}+B}-M_{W} W+E_{W}+D_{W} \nabla^{2} W \\
& =\text { growth }- \text { mortality }+ \text { inflow }+ \text { diffusion. }
\end{aligned}
$$

The process in which living organisms in the sediment actively transport bottom water through their habitats is known as bioirrigation. Bioturbation, the reworking and mixing of the sediment by faunal activity, as well as bioirrigation of benthic fauna substantially affect the mineralization of organic matter in sediments [8]. This transport process is represented by the first term on the right hand side of Eq. (2.1), with $\sigma$ governing its strength. We model this non-local transport process with a simple Fickian-I type function.

The consumption term $F_{V}(U)$ consists of a Holling Type II (Michaelis-Menten) function, which is substrate-dependent and models substrate limitation. Following the model developed by [6], we distinguish between active and dormant microorganisms.

Bacterial dormancy is considered as a survival strategy to adjust to new environments [45]. An overview of different survival strategies of bacteria in natural environments is given 
by Roszak and Colwell [38]. One strategy consists of the transformation of an active to a dormant state. Bacteria in the dormant state function at very low metabolic rates and do not undergo cell division. Another microbial survival mechanism is the formation of spores and cysts. Note that this process occurs on a different time scale and is not taken into consideration here.

In our model, only the active part $g_{V}(V)$ of the total bacteria population $V$ contributes to consumption of $D O C$, therefore we obtain the following mathematical approach for the consumption of $F_{V}(U)$ (analogous for $F_{W}(U)$ ):

$$
F_{V}(U)=V^{a c t} \frac{U}{H_{V}+U}=g_{V}(V) V \frac{U}{H_{V}+U} .
$$

We assume that the ratio between active and dormant organisms is not fixed but varies, since the bacteria are able to switch between both states by self-activation. Active cells may excrete signal molecules, leading to an activation of dormant cells $[11,12]$. The mechanism of self-activation is mathematically described by the function $g_{V}$ (analogous for $W$ ):

$$
g_{V}(V)=\gamma_{V}+\left(1-\gamma_{V}\right) \frac{V}{K_{V}+V}
$$

It is obvious, that $g_{V}(V)$ has to be between zero and one. The parameter $\gamma_{V}$ denotes a certain fraction of the population that is always active. The ratio of active organisms increases as the living conditions are improved. Since the population density is a (fairly) good indicator for the living conditions, the mechanism of self-activation is represented by a Holling type II function, which models the saturation effect of the activation.

The first term of the right hand side of Eqs. (2.2) and (2.3) represents the growth rates of the bacteria populations. The uptake of the organic substrate is multiplied by a Holling type II function, modelling the respiration limited by the electron acceptor $(A$ or $B)$. The parameters $\alpha$ and $\beta$ weight the influence of consumption on bacteria and substrate, respectively. In the following, respiration is summarized in one function for each bacteria species, that we determine by $R_{V}$ and $R_{W}$, respectively:

$$
R_{V}=\alpha_{V} \frac{A}{H_{A}+A}, \quad \text { and accordingly } \quad R_{W}=\alpha_{W} \frac{B}{H_{B}+B} .
$$

Note that, as long as electron acceptor concentrations do not vary in space, $A$ and $B$ are constants. Therefore, each respiration term can then also be represented by a constant. For the spatial system, $A$ and $B$ are depth dependent, thus respiration also varies with the depth of the sediment. Also note that, unlike the electron donor (DOC), the electron acceptor concentrations are independent of microbial activity. This is assumed for the sake of simplicity, as we explicitly focus on the influence of competition for the same substrate. Therefore DOC plays the major role.

Both bacteria population densities are furthermore influenced by a small, but constant inflow term $E_{V / W}$. These mathematical terms have been introduced in order to prevent the sediment from becoming sterile. This would not reflect a realistic biological situation. 
Natural bacteria mortality due to grazers and bacterial viruses is represented by a linear loss term with the mortality rates $M_{V / W}$.

Isotropic transport is described by diffusion of a Fickian-II type. $D_{U}, D_{V}$ and $D_{W}$ are the diffusion coefficients for the substrate and the microorganisms, respectively.

\begin{tabular}{|c|c|c|c|}
\hline parameter & default value/range & units & description \\
\hline$\sigma$ & 2 & $d a y^{-1}$ & strength of bioirrigation [21] \\
\hline$U_{0}$ & 2 & $\mathrm{mmol} / \mathrm{l}$ & $U$ concentration in sea water $[53]$ \\
\hline$\alpha_{V}$ & 0.26 & $d a y^{-1}$ & $V$ growth efficiency $[15]$ \\
\hline$\alpha_{W}$ & 0.3 & $d a y^{-1}$ & $W$ growth efficiency [15] \\
\hline$A$ & 2 & $\mathrm{mmol} / \mathrm{l}$ & concentration of electron acceptor $A$ \\
\hline$B$ & 2 & $\mathrm{mmol} / \mathrm{l}$ & concentration of electron acceptor $B$ \\
\hline$\beta_{V}$ & 0.4 & $d a y^{-1}$ & consumption rate of $V[27]$ \\
\hline$\beta_{W}$ & 0.5 & $d a y^{-1}$ & consumption rate of $W[27]$ \\
\hline$H_{V}$ & 0.01 & $\mathrm{mmol} / \mathrm{l}$ & $\begin{array}{l}V \text { grazing half saturation ( } U \text { up- } \\
\text { take) }[21]\end{array}$ \\
\hline$H_{W}$ & 0.01 & $m m o l / l$ & $\begin{array}{l}W \text { grazing half saturation ( } U \text { up- } \\
\text { take) }[21]\end{array}$ \\
\hline$\gamma_{V}$ & 0.75 & 1 & active part of $V$ \\
\hline$\gamma_{W}$ & 0.6 & 1 & active part of $W$ \\
\hline$K_{V}$ & 2 & $\mathrm{mmol} / \mathrm{l}$ & $V$ activation half saturation \\
\hline$K_{W}$ & 2 & $\mathrm{mmol} / \mathrm{l}$ & $W$ activation half saturation \\
\hline$H_{A}$ & 0.01 & $\mathrm{mmol} / \mathrm{l}$ & half saturation of $A$ respiration [21] \\
\hline$H_{B}$ & 0.1 & $\mathrm{mmol} / \mathrm{l}$ & half saturation of $B$ respiration [21] \\
\hline$M_{V}$ & 0.24 & $d a y^{-1}$ & mortality rate of $V[7]$ \\
\hline$M_{W}$ & 0.24 & $d a y^{-1}$ & mortality rate of $W[7]$ \\
\hline$E_{V}$ & 0.01 & $\mathrm{mmol} / \mathrm{l}$ day & bacterial inflow $V$ \\
\hline$E_{W}$ & 0.01 & $\mathrm{mmol} / \mathrm{l}$ day & bacterial inflow $W$ \\
\hline$D_{U}$ & 0.5 & $c m^{2} / d a y$ & diffusion coefficient of $U[10,21]$ \\
\hline$D_{V}$ & {$[0 \ldots 5]$} & $\mathrm{cm}^{2} / d a y$ & diffusion coefficient of $V$ \\
\hline$D_{W}$ & {$[0 \ldots 5]$} & $\mathrm{cm}^{2} / d a y$ & diffusion coefficient of $W$ \\
\hline
\end{tabular}

Table 1: Dimensional parameters relevant for the three species model (Eqs.(2.1)-(2.3)) and their default values or ranges, respectively. (see main text for explanations.)

$\nabla^{2} \equiv \Delta$ represents the Laplace operator in two space dimensions

$$
\nabla^{2}:=\frac{\partial^{2}}{\partial X^{2}}+\frac{\partial^{2}}{\partial Y^{2}}
$$

where $X$ describes one horizontal dimension and $Y$ describes depth. 
All relevant parameters, their numerical values and brief descriptions are listed in Table 1. Most of the default values are the results of a literature survey and personal communication with [21]. In general, all values are rough estimates based on experimental observations.

The differences between parameters describing the bacterial populations $V$ and $W$ are based on the fact that respiration processes vary in the free energy yield of the individual metabolism. In spite of the electron acceptor concentrations being equal $(2 \mathrm{mmol} / \mathrm{l}$ each), one acceptor (in our case $B$ ) is energetically more favourable. Therefore, microorganism $W$ has an advantage in using electron acceptor $B$. Hence, the parameters for the consumption rates $\beta_{V}, \beta_{W}$ and growth efficiency rates $\alpha_{V}, \alpha_{W}$ for microorganism $W$ have higher values.

Bacteria and nutrient concentrations are usually measured on different scales. For convenience, we express all concentrations in $\mathrm{mmol} / \mathrm{l}$. The concentration of $D O C$ in sea water, $U_{0}=2 \mathrm{mmol} / l$, corresponds to approximately $20 \mathrm{mg} / \mathrm{l}$.

We also assume that species $W$ is more flexible, i.e. more individuals of population $W$ can be in a dormant state. For that reason the default value of $\gamma_{W}$ is smaller than $\gamma_{V}$. Both numbers are chosen rather arbitrarily.

The diffusion coefficients of $V$ and $W$ cannot be estimated easily, since microorganisms either diffuse or move actively by chemotaxis or do not move at all (e.g. in biofilms) [15]. Their diffusion coefficients can therefore be several orders of magnitudes smaller than the diffusion coefficient of $U$, i.e. the substrate $D O C$. In most of the published literature, chemotaxis is taken into account. The effective motility of bacteria through permeable (sandy) porous media lies between $10^{-3}$ and $10^{-1} \mathrm{~cm}^{2} /$ day, see e.g. [3, 36, 35]. As we neglect chemotaxis and focus on less permeable sediments, we assume that the diffusion coefficients of the organisms are in general even smaller, approximately $3-5$ orders of magnitude. This is mainly due to the size difference to $D O C$.

By rescaling, we obtain dimensionless equations with a reduced number of parameters. In particular, we apply the following transformations:

$$
t=\sigma \tau \quad u=\frac{U}{H_{V}} \quad v=V \frac{\beta_{V}}{\sigma H_{V}} \quad w=W \frac{\beta_{W}}{\sigma H_{V}},
$$

and additionally

$$
x^{2}=\frac{X^{2} \sigma}{D_{U}} \quad y^{2}=\frac{Y^{2} \sigma}{D_{U}}
$$

to get a dimensionless diffusive transport. The substitutions yield:

$$
\begin{aligned}
\frac{\partial u}{\partial t} & =\left(u_{0}-u\right)-g_{v}(v) v \frac{u}{1+u}-g_{w}(w) w \frac{u}{h+u}+\nabla^{2} u \\
\frac{\partial v}{\partial t} & =\rho_{v} g_{v}(v) v \frac{u}{1+u}-\mu_{v} v+\epsilon_{v}+\delta_{v} \nabla^{2} v \\
\frac{\partial w}{\partial t} & =\rho_{w} g_{w}(w) w \frac{u}{h+u}-\mu_{w} w+\epsilon_{w}+\delta_{w} \nabla^{2} w .
\end{aligned}
$$

All non-dimensional quantities, their definitions and non-dimensional default values are listed in Table 2. 


\begin{tabular}{|c|c|c|}
\hline non-dimensional quantity & definition & non-dim. default \\
\hline \hline$u_{0}$ & $U_{0} / H_{V}$ & 200 \\
$h$ & $H_{W} / H_{V}$ & 1 \\
$\gamma_{v}$ & {$[0 \ldots 1]$} & 0.75 \\
$\gamma_{w}$ & {$[0 \ldots 1]$} & 0.6 \\
$k_{v}$ & $K_{V} \beta_{V} / \sigma H_{V}$ & 40 \\
$k_{w}$ & $K_{W} \beta_{W} / \sigma H_{V}$ & 50 \\
$\mu_{v}$ & $M_{V} / \sigma$ & 0.12 \\
$\mu_{w}$ & $M_{W} / \sigma$ & 0.12 \\
$\rho_{v}$ & $R_{V} / \sigma$ & 0.1294 \\
$\rho_{w}$ & $R_{W} / \sigma$ & 0.1429 \\
$\epsilon_{v}$ & $E_{V} \beta_{V} / \sigma^{2} H_{V}$ & 0.1 \\
$\epsilon_{w}$ & $E_{W} \beta_{W} / \sigma^{2} H_{V}$ & 0.125 \\
$\delta_{v}$ & $D_{V} / D_{U}$ & {$[0 \ldots 1]$} \\
$\delta_{w}$ & $D_{W} / D_{U}$ & {$[0 \ldots 1]$} \\
\hline
\end{tabular}

Table 2: Non-dimensional parameters relevant for the three species model (2.9) and the associated variable definitions. The non-dimensional defaults are derived according to the parameter estimates presented in Table 1.

Equations (2.9) and the parameters listed in Table 2 are used in the following sections to investigate and analyze the behavior of the system.

\section{Pattern formation in reaction diffusion systems}

In our model, the degradation of chemical substances and the relevant transport processes are specified in a system of partial differential equations, i.e. a 3-component reaction diffusion system (RDS). In order to qualitatively investigate this 3-component RDS, we apply methods from dynamical systems theory. Therefore, we first introduce a general 3-component RDS and analyze it in terms of pattern formation. We show that the interaction between reaction and transport processes can lead to the spatial destabilization of a previously homogeneous stable steady state. Moreover, we derive the general condition for diffusion-induced stationary patterns to evolve.

\subsection{The Turing mechanism}

In his seminal paper [49], Turing demonstrated theoretically that a system of two reacting and diffusing chemicals can spontaneously evolve spatially heterogeneous patterns from an initially uniform state in response to infinitesimal perturbations. Remarkably, he showed that under certain conditions diffusion can drive a chemical system to instability, leading 
to spatially inhomogeneous patterns. A reaction diffusion system exhibits a diffusion-driven instability, a so-called Turing instability, if the homogeneous steady state is locally stable with respect to small perturbations in the absence of diffusion, but unstable to small perturbations for the full RDS [32]. Satnoianu et al. [41] have derived mathematical conditions on the kinetics of an $n$-dimensional system under which diffusion-driven instabilities may arise. In the following Section 3.2, we specify these findings for a system of $n=3$ reacting and diffusing species.

\subsection{General conditions for diffusion-driven instability}

Consider a coupled system of 3 species (or concentrations of chemicals) which interact nonlinearly and may diffuse differently. Mathematically, this problem is described by the equations

$$
\frac{\partial u_{j}}{\partial t}=f_{j}(\mathbf{u})+D_{j} \Delta u_{j}, \quad j=1,2,3
$$

with given non-negative initial concentrations

$$
u_{j}(\mathbf{x}, 0)=u_{j, 0}(\mathbf{x}) .
$$

Here, $\mathbf{u}=\left(u_{1}, u_{2}, u_{3}\right)$ is the vector of concentrations of the interacting species at position $\mathbf{x}$ and time $t . D_{j}$ is the respective diffusion coefficient and $f_{j}(\mathbf{u}), j=1,2,3$ represent the local reaction terms.

In order to solve the problem, we require appropriate boundary conditions. Because we want to omit any effects of forcing, we choose periodic boundary conditions. We assume that Eq.(3.10) possesses a stable stationary homogeneous solution $\mathbf{u}=\mathbf{u}^{*}$ and investigate its stability with respect to spatial perturbations having a wavenumber $k$.

By means of linear stability analysis, we obtain a stability criterion, that holds for any homogeneous equilibrium of a RDS (for details, see e.g. [32]). In particular, we derive the stability of the RDS with respect to perturbations of wavenumber $k^{2}$ by considering the eigenvalues of the matrix $\mathbf{C}$ :

$$
\begin{gathered}
\mathbf{C}=\mathbf{A}-k^{2} \mathbf{D}=\left(c_{i j}\right)=\left(a_{i j}-k^{2} D_{j} \delta_{i, j}\right), \quad i, j=1,2,3 \\
\text { with } \mathbf{A}=\left(a_{i j}\right)=\left.\frac{\partial f_{i}}{\partial u_{j}}\right|_{\mathbf{u}=\mathbf{u}^{*}}
\end{gathered}
$$

where $\delta_{i, j}$ is the Kronecker symbol and $\mathbf{A}$ is the Jacobian matrix associated with the local reaction part of the system. For $k^{2}=0$, the Jacobian of the RDS is equivalent to the one associated with the local system. With increasing $k^{2}$, the real parts of one or two eigenvalues of $\mathbf{C}$ may become positive, meaning that the homogeneous steady state may become (Turing) unstable.

The number of eigenvalues changing the sign of their real parts give the type of bifurcation: A Turing instability can occur via a Hopf bifurcation, in which two complex conjugate eigenvalues of the Jacobian cross the imaginary axis, or a saddle-node bifurcation, which 
is in general characterized by the presence of a zero eigenvalue of the Jacobian matrix $\mathbf{C}$ [17]. In the following, we concentrate on the latter case, as in our model the loss of stability corresponds to a saddle-node bifurcation.

Thus, we now derive the conditions on the diffusion coefficients $D_{j}$ and the kinetics matrix A for which there exists at least one eigenvalue for $\mathbf{C}$ with positive real part.

We get the eigenvalues $\lambda$ by solving

$$
\operatorname{det}(\mathbf{C}-\lambda \mathbf{I})=0 .
$$

If all eigenvalues of a 3-dimensional system exhibit negative real parts, the product of all eigenvalues will also be negative. Since the determinant of a matrix equals the product of all eigenvalues [48], the loss of stability is associated with the change of sign of $\operatorname{det} \mathbf{C}$. The condition for the steady state to become Turing unstable therefore reads:

$$
\operatorname{det}(\mathbf{C})=\operatorname{det}\left(\mathbf{A}-k^{2} \mathbf{D}\right)=\operatorname{det}\left(a_{i j}-D_{j} k^{2} \delta_{i, j}\right) \geq 0 .
$$

The characteristic equation is a continuous function and has the form of a cubic polynomial with respect to $k^{2}$ :

$$
\operatorname{det} \mathbf{C}\left(k^{2}\right)=m_{3} k^{6}+m_{2} k^{4}+m_{1} k^{2}+m_{0}>0 .
$$

Straight forward calculations yield

$$
\begin{aligned}
m_{3} & =-D_{1} D_{2} D_{3}=-\prod_{j} D_{j}=-\operatorname{det}(\mathbf{D}) \\
m_{2} & =a_{11} D_{2} D_{3}+a_{22} D_{3} D_{1}+a_{33} D_{1} D_{2} \\
& =\sum\left(a_{i i} D_{j} D_{l}\right)=\sum\left(\Delta_{i} D_{j} D_{l}\right) \\
m_{1} & =-\left[D_{1}\left(a_{22} a_{33}-a_{23} a_{32}\right)+D_{2}\left(a_{33} a_{11}-a_{31} a_{13}\right)+D_{3}\left(a_{11} a_{22}-a_{12} a_{21}\right)\right] \\
& =-\left[D_{1} \Delta_{23}+D_{2} \Delta_{31}+D_{3} \Delta_{12}\right]=-\sum \Delta_{i j} D_{l} \\
m_{0} & =\operatorname{det}(\mathbf{A}),
\end{aligned}
$$

where $1 \leq i, j, l \leq 3$, with $i, j, l$ being distinct and rotating cyclically. $\Delta_{i j}$ and $\Delta_{i}$ correspond to the determinants of the 2- and 1-dimensional subsystems of $\mathbf{A}$, respectively.

Since the system is assumed to be locally stable, all eigenvalues of $\mathbf{A}$ have negative real parts and $m_{0}=\operatorname{det}(\mathbf{A})<0$ and therefore $\operatorname{det} \mathbf{C}\left(k^{2}=0\right)<0$. Since $m_{3}<0$ due to the positive diffusion coefficients, it is also apparent from Eq. (3.14) that

$$
\lim _{k^{2} \rightarrow \infty} \operatorname{det} \mathbf{C}\left(k^{2}\right)=-\infty .
$$

It is therefore necessary for the existence of an eigenvalue $\lambda$ with positive real part that $\operatorname{det} \mathbf{C}\left(k^{2}\right)$ exhibits a maximum for a specific $k_{\max }^{2}>0$ and sufficient that $\operatorname{det} \mathbf{C}\left(k_{\max }^{2}\right)>0$. 
The specific $k_{\max }^{2}$ satisfying the necessary condition must be a solution of

$$
\frac{\partial \operatorname{det} \mathbf{C}\left(k^{2}\right)}{\partial k^{2}}=3 m_{3} k^{4}+2 m_{2} k^{2}+m_{1}=0
$$

Eq. (3.16) possesses two solutions. It is required that the second derivative of $\operatorname{det}(\mathbf{C})$ with respect to $k^{2}$ has to be negative, hence only one of the solutions of (3.16) is relevant. As $m_{3}$ is always negative due to the positive diffusion coefficients, this solution reads

$$
k_{\max }^{2}=-\frac{m_{2}}{3 m_{3}}-\frac{1}{3 m_{3}} \sqrt{m_{2}^{2}-3 m_{3} m_{1}}>0 .
$$

The necessary condition $k_{\max }^{2}>0$ can only be fulfilled if

$$
m_{2}>0 \quad \text { or } \quad m_{1}>0 .
$$

$m_{2}>0$ corresponds to the existence of $\Delta_{i}=a_{i i}>0$ for at least one $i \in\{1,2,3\}$, i.e. one diagonal element of $\mathbf{A}$ must be positive.

For $m_{1}>0$ it is necessary, that there are $1 \leq i, j \leq 3$ such that $\Delta_{i j}<0$, which means that one 2-dimensional subsystem $\Delta_{i j}$ of $\mathbf{A}$ needs to be unstable.

Two different possible cases of Turing bifurcations can therefore be distinguished [41]:

1. The case where $\Delta_{i}=a_{i i}>0$ for at least one $i, 1 \leq i \leq 3$, is defined as a Turing bifurcation of the first type $(I)$.

2. A Turing bifurcation of the second type (II) is defined as the case where there are $1 \leq i, j \leq 3$, such that $\Delta_{i j}<0$, and the bifurcation is not of the first type.

In other words, the dimension of the unstable subsystem of the Jacobian A determines the type of the Turing bifurcation. An unstable subsystem is also referred to as an activator, whereas a stable subsystem is known as an inhibitor [32, 41]. The presence of an activator is necessary but not sufficient for the diffusive destabilization of the system.

The sufficient condition can only be fulfilled by finding appropriate wavenumbers and diffusion coefficients. It has been demonstrated by Qian and Murray [39] that the following criteria exist to enable reaction systems with three species to exhibit diffusion-driven instability: The sufficient condition is that either the largest diagonal element of $\mathbf{C}$ is greater than zero $\left(a_{i i}>0\right)$ with the corresponding diffusion coefficient $D_{i}$ being very small, or the smallest determinant $\Delta_{i j}$ is smaller than zero with the corresponding diffusion coefficient $D_{l}$ being very large.

In the following, these more general considerations will be applied to our model system, describing the interaction of two bacteria species with their substrate.

\section{Model analysis}

We now investigate the qualitative behavior of the reaction diffusion system (Eq. (2.9)) in order to understand the mechanism of pattern formation for this specific model of two 
competing microbial species. We first study the local model, meaning that diffusive transport is neglected. Thereby special attention is paid to the parameter describing respiration. Afterwards, we investigate the whole reaction diffusion system by analyzing the spatial model and deriving the conditions for Turing instabilities.

\subsection{Analysis of the local model}

The local model consists of Equations (2.9), not regarding any spatial derivatives and assuming spatially constant electron acceptor concentrations. Thus, the diffusion terms are neglected and the respiration function can be considered as a fixed value (cf. Eq. (2.6)):

$$
\begin{aligned}
\frac{\partial u}{\partial t} & =\left(u_{0}-u\right)-g_{v}(v) v \frac{u}{1+u}-g_{w}(w) w \frac{u}{h+u} \\
\frac{\partial v}{\partial t} & =\rho_{v} g_{v}(v) v \frac{u}{1+u}-\mu_{v} v+\epsilon_{v} \\
\frac{\partial w}{\partial t} & =\rho_{w} g_{w}(w) w \frac{u}{h+u}-\mu_{w} w+\epsilon_{w} .
\end{aligned}
$$

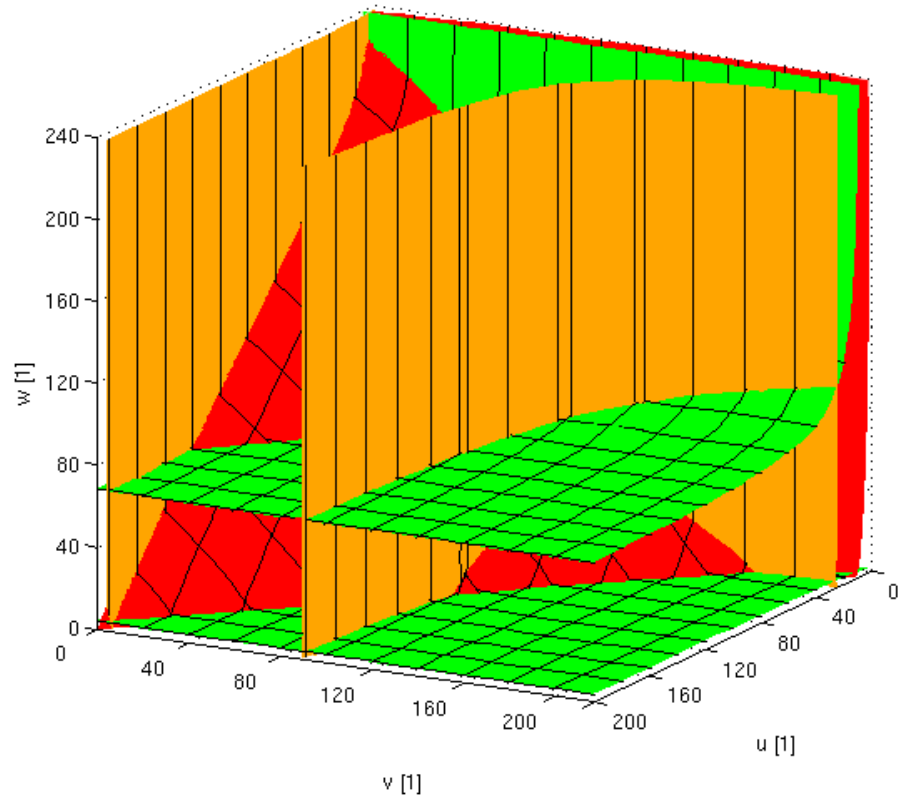

Figure 2: Nullclines for the default parameterisation (see Table 2) in three dimensional state space: $\frac{d u}{d t}=0$ is plotted as a red surface, $\frac{d v}{d t}=0$ is plotted orange and the green surface corresponds to $\frac{d w}{d t}=0$. The black solid lines illustrate $u$ - and $v$-isoclines.

To study the steady states (i.e. the stationary solutions) of the local model, we consider those states in which the time derivatives in Eqs. (4.19) vanish. Although the identification of 
the steady states of our general system is troublesome due to the complex consumption terms, we have been able to determine the general steady states analytically by using computer algebra. Nevertheless, we omit to write down the equilibria's coordinates in general terms, because the particular expressions are far too complex to tell us much about the system's behavior. It is possible to prove the existence of (at least) one positive equilibrium of system (4.19) [42]. However, the explicit calculation of nullclines and consequently steady states is for all intents and purposes sensible for a specific parametrization. In particular, we focus on our biologically relevant default parameters presented in Table 2.

In Figure 2, the nullclines of the local system (4.19) for our default parametrization are illustrated in 3-dimensional state space ${ }^{2}$.

The $u, v$ and $w$ nullclines are plotted in the colors red, orange and green, respectively. The solid black lines denote both, $u$ - and $v$-isoclines. We see that the nullclines intersect in five points in state space, therefore the system exhibits five steady states. Explicit calculation of the steady states and linear stability analysis yield the following coordinates and characteristics:

$$
\begin{array}{ll}
\mathbf{u}_{1}^{*}=\left(\begin{array}{c}
193.52 \\
5.04 \\
4.12
\end{array}\right): \text { attractor } & \mathbf{u}_{4}^{*}=\left(\begin{array}{c}
138.32 \\
4.98 \\
69.92
\end{array}\right) \text { : saddle } \\
\mathbf{u}_{2}^{*}=\left(\begin{array}{c}
28.84 \\
182.95 \\
3.76
\end{array}\right): \text { attractor } & \mathbf{u}_{5}^{*}=\left(\begin{array}{c}
106.10 \\
99.37 \\
4.06
\end{array}\right) \text { : saddle } \\
\mathbf{u}_{3}^{*}=\left(\begin{array}{c}
9.13 \\
3.30 \\
225.61
\end{array}\right) \text { : attractor } &
\end{array}
$$

The three coexisting stable equilibria, i.e. attractors, $\mathbf{u}_{1}^{*}, \mathbf{u}_{2}^{*}$ and $\mathbf{u}_{3}^{*}$ are located close to the margins of the positive octant, meaning that in those solutions one variable, i.e. either one bacteria species or the substrate, is quantitatively clearly dominating the two others. The dominance of one bacteria species is due to the competition exclusion principle. This principle would cause the extinction of the weak species in favor of the dominant one. In our specific model, however, the constant inflow $\epsilon_{v, w}$ assures that the populations of microorganisms cannot become extinct and thus the sediment cannot become sterile. This assumption implicates that the populations of microorganisms cannot outcompete one another completely. As a result, both populations do coexist with one species clearly dominating.

Let us now investigate the behavior of the biological model with respect to changes of selected ecosystem-specific parameters ${ }^{3}$. In particular, we focus on the system's sensitivity to the respiration terms. This investigation provides the basis for the simulations presented in Section 5, where we consider depth-dependent electron acceptor concentrations and hence depth-dependent respiration.

\footnotetext{
${ }^{2}$ The Figure was made using a software based on adaptive triangulation developed by [46].

${ }^{3}$ The model analysis was carried out using the continuation and bifurcation analysis software 'CONTENT' $[24]$.
} 

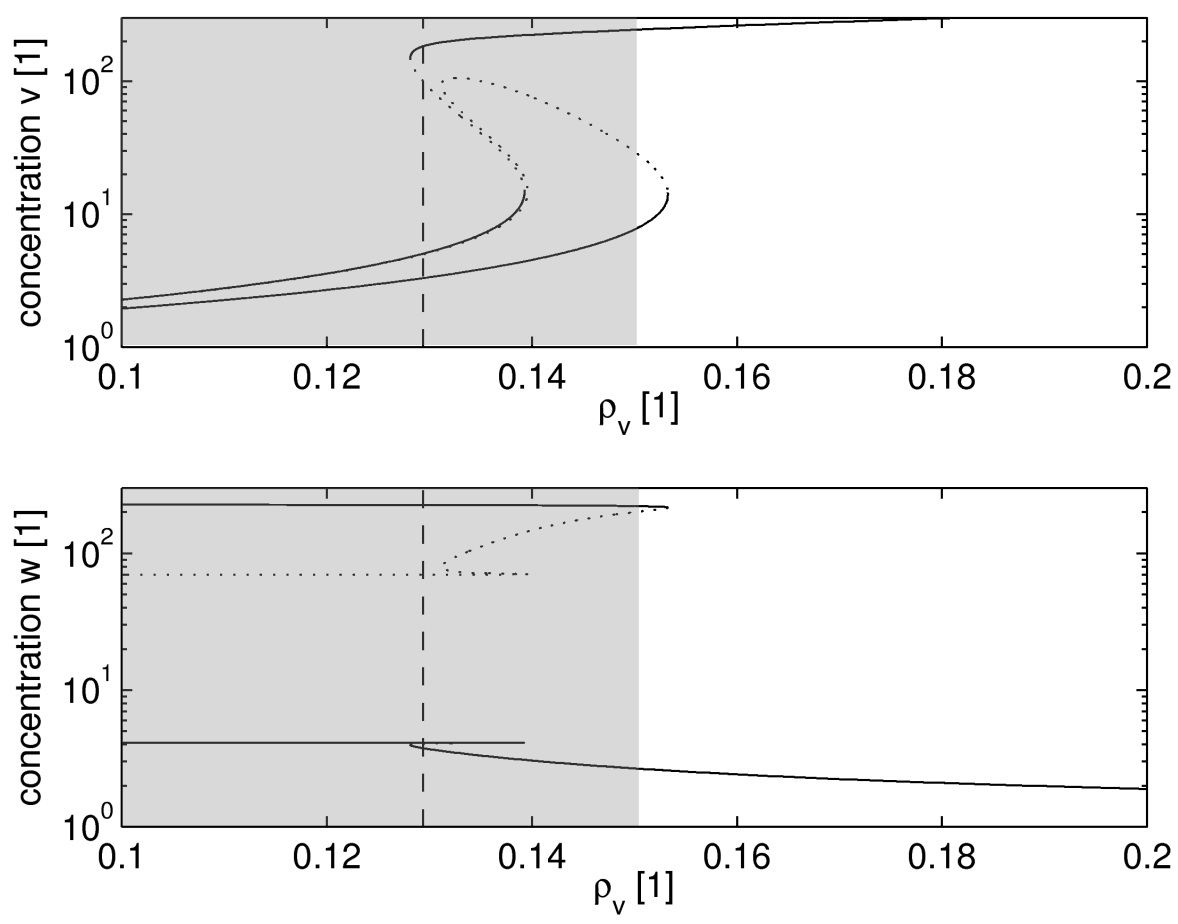

Figure 3: Dependence of the species concentration $v$ and $w$ on $\rho_{v}$; solid curves: stable steady states; dotted curves: unstable steady states; dashed line: default value $\rho_{v}=0.1294$ and $\rho_{w}=0.1429$, respectively; all default parameter values are taken from Table 2 . The role of the shaded area will be explained in Sec. 5 .

\subsection{Sensitivity to the respiration term}

The uptake of organic substrate, and therefore the bacteria growth, is limited by electron acceptor $(A$ or $B)$ respiration (see Eqs. (2.6)). In the local (e.g. default) case, $A$ and $B$ are constants. Therefore, each respiration term is represented by a parameter.

Figure 3 illustrates the long-term behavior of the species concentrations $v$ and $w$ with respect to the respiration term $\rho_{v}$ : All transitions in the system's stability correspond to saddle-node bifurcations. We see that, apart from such 'tristable' parametrizations like our default set, there exist parameter ranges where the system is bistable and where only one stable equilibrium can be found. In Figure 4 the system's behavior is illustrated in parameter space. Here, the solid lines illustrate the saddle-node bifurcations, whereas the dashed lines refer to the default values. We see that the plots presented in Figure 3 are illustrations of the system's behaviour for parameter variation along the dashed lines in Figure 4.

It appears that the system is multistable for a broad range of values $0.1<\rho_{v}, \rho_{w}<0.2$. In particular, for $0.129 \lesssim \rho_{v} \lesssim 0.139$ and $0.134 \lesssim \rho_{w} \lesssim 0.164$ the system exhibits three stable coexisting equilibria. At $\rho_{v}=0.129$ the stable solution with a high abundance of $v$ 


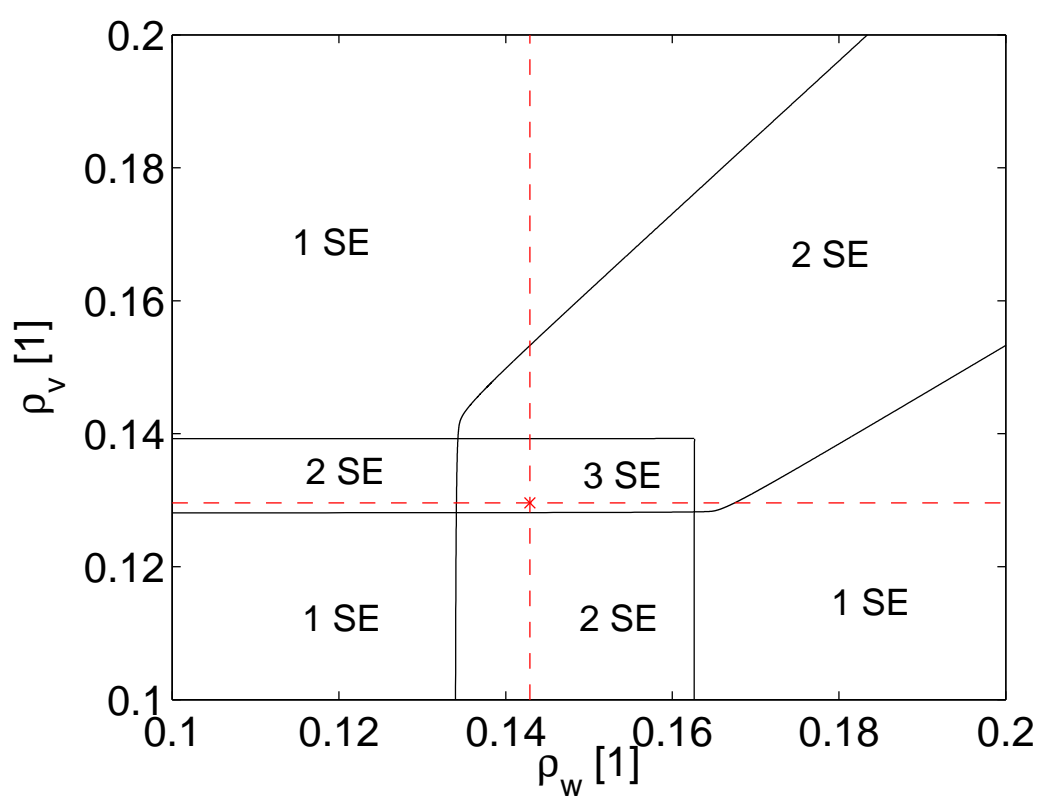

Figure 4: Bifurcation diagram; solid curves correspond to a saddle-node bifurcation; dashed line: default value of $\rho_{v}$ and $\rho_{w}$, respectively; SE: stable equilibrium (equilibria); parameters from Table 2.

merges with an unstable solution in state space, i.e. a saddle node bifurcation occurs. For $\rho_{v}<0.129$ no stable solution with a high concentration of species $v$ exists. Therefore, we conclude that the corresponding living conditions are not good enough for $v$ to successfully compete with $w$. On the other hand, if the living conditions for $v$ are better than with our default parametrization, i.e. $\rho_{v}>0.139$, the stable solution with a high concentration of $w$ loses its stability due to a saddle node bifurcation. Here, species $v$ dominates the other species $w$.

For a variation of $\rho_{w}$ we see an equivalent situation.

As an example of how the degree of nonlinearity influences the behavior of the system, Figure 5 shows the dependency of stability (the saddle-node bifurcations plotted as solid lines) on $\gamma_{v}$ as well as on $\rho_{v}$. Again, the dashed line corresponds to the default parametrization. The parameters $\gamma_{v}$ and $\gamma_{w}$ control the influence of the activation mechanism on the microorganism's population densities. If $\gamma$ is very small, the population may be almost entirely activated by a few individuals of the corresponding species. If $\gamma$ is very large, the part of the population which is always actively contributing to the consumption is large, too. From the mathematical point of view, the increase of $\gamma$ coincides with the decrease of the nonlinearity of the system. For $\gamma_{v}=1$ the associated equations are linear in $v$. In that case the system exhibits two stable equilibria for $\rho_{v}<0.129$. For higher values of $\rho_{v}$, we only get one stable solution. At $\gamma_{v} \simeq 0.93$ we see a cusp, at values $\gamma_{v}$ smaller than this critical value the system can exhibit three stable equilibria for certain parametrizations.

We see that the parameter range, which allows for multistability, increases with decreasing 


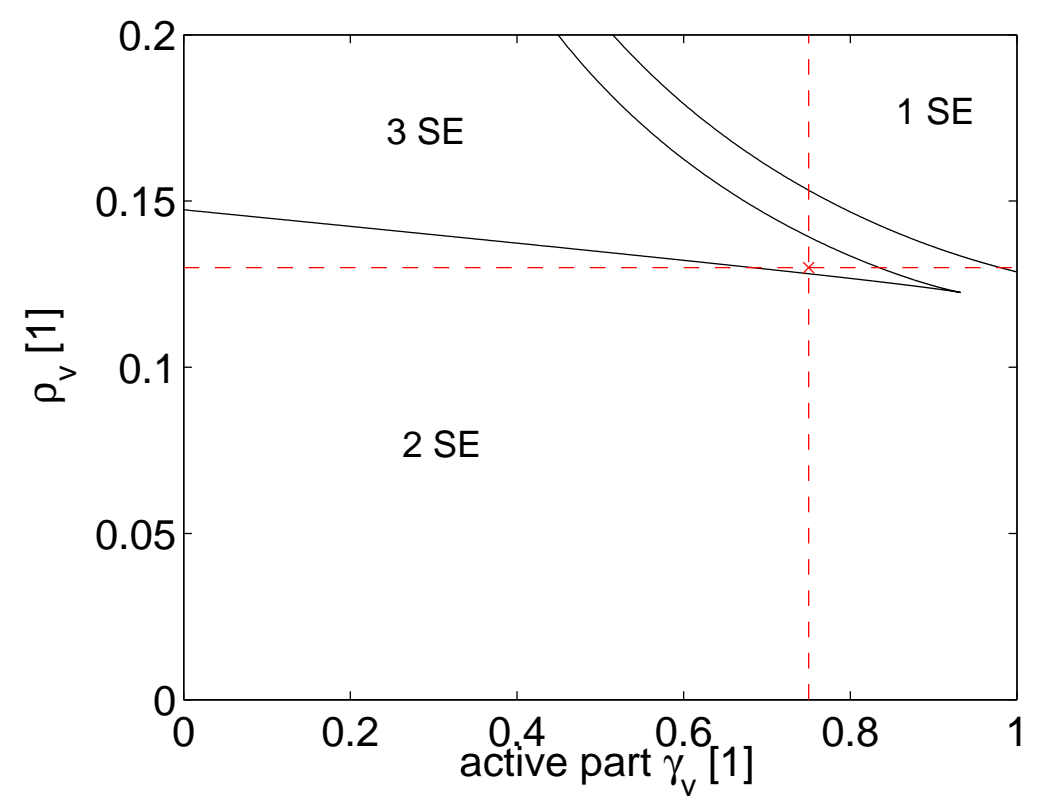

Figure 5: Bifurcation diagram: solid curve: saddle-node bifurcation; dashed line: default value of $\gamma_{v}=0.75$ and $\rho_{v}=0.1294$, respectively; SE: stable equilibrium (equilibria); default parameter values from Table 2

parameter $\gamma_{v}$ and therefore increasing nonlinearity.

\subsection{Analysis of the spatial model}

To study whether diffusive transport can lead to spatially inhomogeneous patterns of chemicals and microorganisms in the sediment, we investigate our model on a two dimensional (2D) spatial domain. Beside the reaction part, we also take diffusion into account. The model (see Eqs. (2.9)), is now analyzed in terms of pattern formation. In this section we consider the model without any non-homogeneous forcing, since this ensures that the patterns are not imposed by external forcing. We assume that the biological, chemical and physical conditions do not depend on space and that there are no non-homogeneous effects due to the boundary conditions. This can be achieved by choosing periodic boundary conditions.

In contrast to the local model, we dispose our spatial model (2.9) on a two-dimensional domain, so that steady state solutions are 2D-functions, too. In this section, these solutions are homogeneous (e.g. spatially constant) functions. As diffusive flows are driven by spatial gradients, there are no fluxes in case of the homogeneous distribution. Hence, the spatially homogeneous solutions can be obtained from the solutions of the local model, which we have discussed in the previous Section 4.1.

Let us now discuss the stability of the homogeneous steady state with respect to spatially inhomogeneous perturbations. In Section 3.2, we have derived the general conditions for a so-called Turing instability to occur in a system of three reacting and diffusing species. 
We have identified the necessary and sufficient conditions, under which a steady state can undergo a Turing bifurcation. Now, we check these conditions for our specific model.

In our specific non-dimensional model the components of the Jacobian associated to the local system (4.19) exhibit the following signs:

$$
\mathbf{A}=\left(\begin{array}{ccc}
a_{11} & a_{12} & a_{13} \\
a_{21} & a_{22} & a_{23} \\
a_{31} & a_{32} & a_{33}
\end{array}\right)=\left(\begin{array}{ccc}
- & - & - \\
+ & +/- & 0 \\
+ & 0 & +/-
\end{array}\right)
$$

We see that a positive diagonal element, i.e. an unstable one-dimensional subsystem, can exist $\left(a_{22}\right.$ or $\left.a_{33}>0\right)$. However, an unstable two-dimensional subsystem $\Delta_{i j}<0$ without any positive diagonal entries cannot. Therefore, the Turing bifurcation in our specific system will always be of the first type.

We can generalize this finding: Since the sign of the component $a_{i j}$ of the Jacobian determines the character of the interaction between the species $i$ and $j$, it is in general typical for predator-prey-systems that they do not exhibit any unstable subsystems of a dimension $n>1$. This is true for food chains of any dimension. Also in food webs, in which species of one trophic level do not interact via direct competition or mutualism, diffusion induced pattern formation is always of Turing type I. Thus it presumes autocatalytic growth of at least one species. Our specific case treats a (simple) food web without any direct interaction between the bacteria species (which are on the same trophic level (i.e. the predators)). We conclude, that the system can not lose stability due to a Turing type II bifurcation. In the following we hence focus on the Turing type I bifurcation.

Additionally, we note that in our system the species $u$ (representing the substrate $D O C$ ) is the inhibitor $\left(a_{11}<0\right)$, whereas both populations of microorganisms can be the activating species, as $a_{22}$ and $a_{33}$ can be positive. Whether both, one or none of the species can act as the activator, depends on the parametrization. Straight forward calculation yields

$$
\begin{aligned}
a_{22} & =\rho_{v} g_{v}(v) \frac{u}{1-u}+\rho_{v} v \frac{u}{1-u}\left(1-\gamma_{v}\right) \frac{k_{v}}{\left(k_{v}+v\right)^{2}}-\mu_{v} \\
& =-\rho_{v} a_{12}-\mu_{v} .
\end{aligned}
$$

Therefore, the condition $a_{22}>0$ can only be fulfilled, if

$$
-\rho_{v} a_{12}>\mu_{v}
$$

Analogously, we find $a_{33}>0$, if

$$
-\rho_{w} a_{13}>\mu_{w}
$$

with $a_{12}$ and $a_{13}$ always being negative. This shows that both species of bacteria can be the activator due to their ability of self-activation. Active bacteria can activate other dormant organisms of their population by excreting signal molecules. Hence, both populations can possibly grow autocatalytically. The presence of an activator is necessary but not sufficient for the diffusive destabilization of the system. The necessary condition has been obtained 
independently of $k$ and $\mathbf{D}$. The sufficient condition for a Turing type I bifurcation to occur, requires the activator to be less mobile than the inhibitor. Therefore, it can only be fulfilled by finding appropriate wavenumbers and diffusion coefficients.

Since det $\mathbf{C}$ is cubic in $k^{2}$, negative for $k^{2}=0$ and approaches minus infinity for $k^{2} \rightarrow \infty$ (see Section 3.2), we find that a change of stability occurs, if $\operatorname{det} \mathbf{C}$ exhibits a maximum with positive value. The range of instability for a locally stable equilibrium is then limited by two critical wavenumber values $\left(k_{-}^{2}>0\right.$ and $\left.k_{+}^{2}>0\right)$. We refer to this range of instability as the Turing space. The size of this range not only depends on the kind of equilibrium, but also on the diffusion coefficients.

For illustration, let us consider our default parametrization again (see Table 2). In addition, we choose the ratios of diffusion coefficients to be $\delta_{v}=\delta_{w}=10^{-5}\left(\delta_{u}\right.$ is rescaled to one). The system exhibits three homogeneous stable equilibria $S E_{1}, S E_{2}$ and $S E_{3}$ with associated Jacobian matrices A:

$$
\begin{aligned}
& S E_{1}=\left(\begin{array}{c}
193.52 \\
5.04 \\
4.12
\end{array}\right), \quad \mathbf{A}\left(S E_{1}\right) \quad=\left(\begin{array}{ccc}
-1.000 & -0.799 & -0.655 \\
+0.001 & -0.017 & 0 \\
+0.001 & 0 & -0.027
\end{array}\right) \\
& S E_{2}=\left(\begin{array}{c}
28.84 \\
182.95 \\
3.76
\end{array}\right), \quad \mathbf{A}\left(S E_{2}\right) \quad=\left(\begin{array}{ccc}
-1.199 & -0.959 & -0.623 \\
+0.025 & +0.004 & 0 \\
+0.001 & 0 & -0.030
\end{array}\right) \\
& S E_{3}=\left(\begin{array}{c}
9.13 \\
3.30 \\
225.61
\end{array}\right), \quad \mathbf{A}\left(S E_{3}\right) \quad=\left(\begin{array}{ccc}
-3.064 & -0.710 & -0.890 \\
+0.003 & -0.028 & 0 \\
+0.291 & 0 & +0.007
\end{array}\right)
\end{aligned}
$$

The top left panel of Fig. 6 shows the determinants of the Jacobian det $\mathbf{C}$ of $S E_{2}$. In the other three panels of Fig. 6, the maximum real parts of the eigenvalues $\operatorname{Re}(\lambda)$ of all locally stable equilibria $S E_{2}, S E_{1}$ and $S E_{3}$ are plotted versus the wavenumber $k^{2}$.

Influenced by a homogeneous perturbation, the equilibria react like their counterparts in the local model. Looking at the intersection with the $\left(k^{2}=0\right)$-axis we see, that all equilibria are stable with respect to homogeneous perturbations, since all eigenvalues of $\mathbf{C}$ have negative real parts.

In the top panels of Fig. 6, we present the typical situation of a Turing instability. With respect to homogeneous perturbations, $S E_{2}$ is stable. Its associated Jacobian (see Eq. (4.25)) shows that species $v$ is growing autocatalytically and hence it is the activator. With increasing wavenumber $k^{2}$ one (maximum) eigenvalue and simultaneously the determinant det $\mathbf{C}$ changes its sign. This critical wavenumber $k_{-}^{2} \approx 10$ limits a range of perturbations inducing the destabilization of the homogeneous steady state. This range is bounded by $k_{+}^{2} \approx 799$. In this Turing space it is possible for the homogeneous equilibrium $S E_{2}$ to lose stability due to diffusive transport. Therefore, we refer to $S E_{2}$ as being Turing unstable. Concerning the third stable equilibrium $S E_{3}$ (Fig. 6, bottom left), the situation is equivalent but the size 

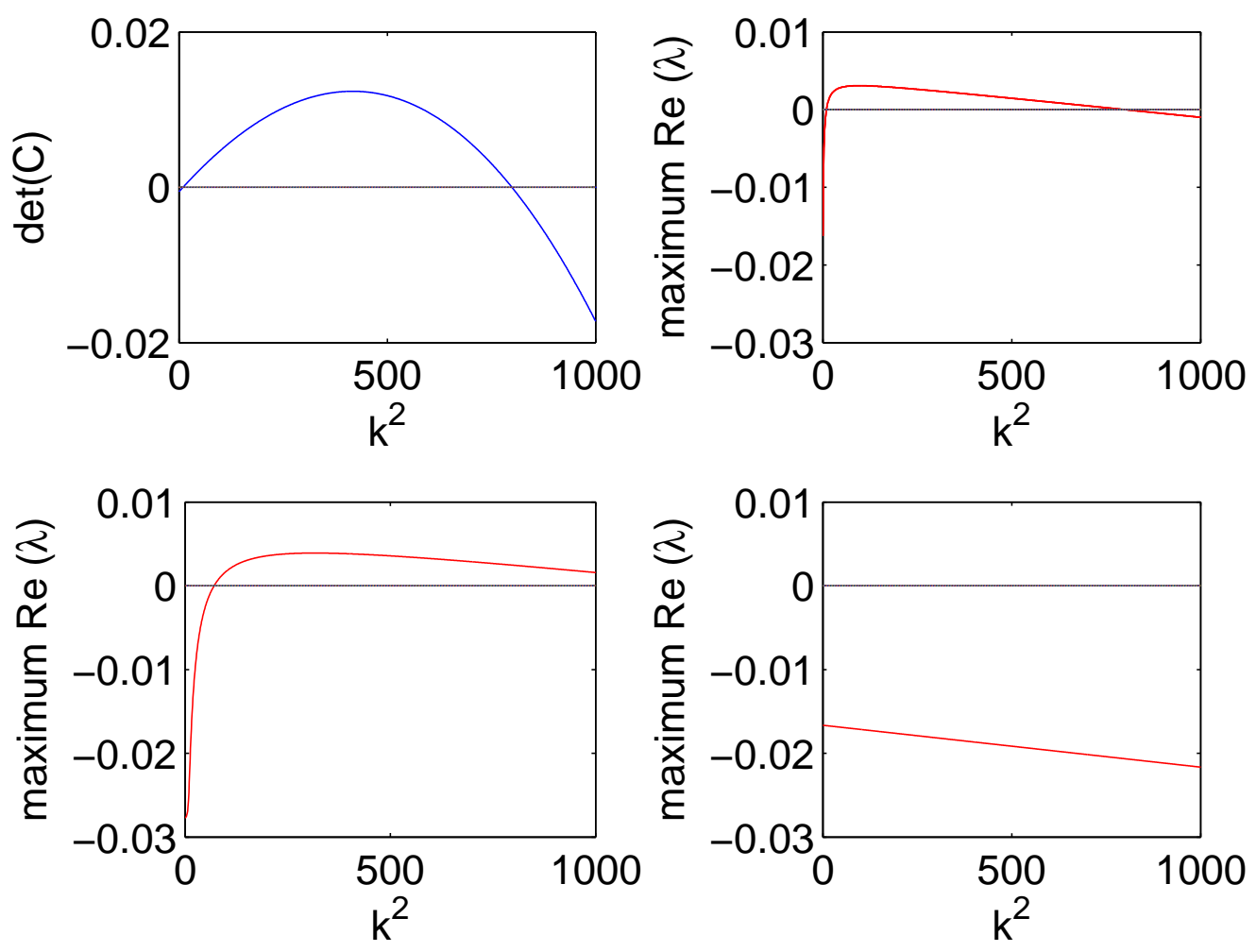

Figure 6: Stability analysis of stable equilibria (see Eqs. (4.24)-(4.26)): the determinant of the Jacobian det $\mathbf{C}$ of $S E_{2}$ (top left panel) and the maximum real parts of all eigenvalues $\operatorname{Re}(\lambda)$ of all locally stable equilibria are plotted over the wavenumber $k^{2}$ (top right: $S E_{2}$; bottom left: $S E_{3}$; bottom right: $S E_{1}$ ); default parametrization from Table 2 and $\delta_{v}=\delta_{w}=10^{-5}$.

of the Turing structures is different. The bottom right panel of Fig. 6 shows that $S E_{1}$ is unconditionally stable, meaning that perturbations of any wavelength will be damped out. This is also apparent when taking a look at the associated Jacobian in Eqs. (4.24): the necessary condition, the existence of an unstable subsystem, is not fulfilled, since all diagonal entries are negative. Hence, the function $\operatorname{det} \mathbf{C}\left(k^{2}\right)$ does not exhibit a maximum, but is monotonically decreasing.

The range of the Turing space does not only depend on the wavenumber $k^{2}$, but also on the diffusion coefficients. With $\delta_{v}$ and $\delta_{w}$ being one order of magnitude larger (not shown), only equilibrium $S E_{2}$ is Turing unstable. By contrast equilibrium $S E_{3}$ is unconditionally stable. In mathematical terms this means: Although the function $\operatorname{det} \mathbf{C}\left(k^{2}\right)$ evaluated at $S E_{3}$ has a positive maximum, the values of this function are always negative for $k^{2}>0$. To conclude, $\operatorname{det} \mathbf{C}$ and $R e(\lambda)$ as functions of $k^{2}$ vary for various diffusion coefficients $\delta_{v, w}$. For spatial patterns to evolve, two conditions must hold simultaneously. First, the spatially uniform steady state must be stable with respect to small perturbations in the absence of diffusion, i.e all $\lambda\left(k^{2}\right)$ have $\operatorname{Re} \lambda\left(k^{2}=0\right)<0$. Second, only patterns of a certain spatial 
extent, that is patterns within a definite range of wavelengths corresponding to wavenumbers $k$, can begin to grow $\left(\operatorname{Re} \lambda\left(k^{2} \neq 0\right)>0\right)$. That means the system will amplify patterns of a particular spatial extent, when excited by random fluctuations, which are always present in biological systems.

\section{Pattern formation in sediments}

The occurrence of a Turing instability only tells us, that a spatially homogeneous distribution of substrate and microorganisms is unstable with respect to certain perturbations. To study how and what kind of patterns evolve in our sediment model, we perform several series of computer simulations. In this section, we present a choice of evolving patterns corresponding to different initial and boundary conditions, as well as external forcing of different types, to account for different biological conditions. We simulate the dynamics of the concentration of substrate and populations of microorganisms on a two-dimensional horizontal-vertical model domain, that has a length of $2.6 \mathrm{~cm}$ and a depth of $2 \mathrm{~cm}$.

\subsection{Homogeneous supply of electron acceptors}

In the first series of simulations, we consider the model without any non-homogeneous forcing. We assume that the biological, chemical and physical conditions do not depend on space. Hence, the structure evolving is not imposed by any external forcing, but only due to the interaction of local reaction and diffusion. Moreover, the initial homogeneous $\mathbf{u}^{*}-$ distributions are stationary, with $\mathbf{u}^{*}=\left[u_{s}, v_{s}, w_{s}\right]$ being an attracting state in the local model which is potentially Turing-unstable. Furthermore, we assume that our domain is infinite and thus we choose periodic boundary conditions, as this premise ensures that there are no non-homogeneous effects due to boundary conditions. We start each simulation at the homogeneous equilibrium $\mathbf{u}^{*}$ and apply small random perturbations.

In the previous Section 4.3, we have seen that our system (see Table 2) exhibits two Turing-unstable equilibria in the default parametrization, if the diffusion coefficients of both bacteria are equal $\delta_{v}=\delta_{w}=10^{-5}$. As two Turing-unstable equilibria exist, we accomplish two simulations with two different initial homogeneous distributions $S E_{2}$ and $S E_{3}$ (see Eqs. (4.25 and 4.26)), respectively. We neglect equilibrium $S E_{1}$ as an initial distribution, as it is unconditionally stable (see Section 4.3). It cannot be destabilized by perturbations and hence the species remain homogeneously distributed.

Figure 7 shows the stationary patterns that emerge in the distribution of the substrate $u$ (top left panel) and of the two bacteria population densities $v$ (top right) and $w$ (bottom) with the initial condition distribution set on the spatially homogeneous but randomly perturbed equilibrium $S E_{2}$ (see Eqs. (4.25)), which exhibits a high concentration of the dominant, activating species $v$. In this case the default parametrization leads to a spatial distribution of isolated zones with high abundance of the activating species $v$. These patterns can be regarded as 'hot spots'. The distributions corresponding to substrate and the second population of microorganisms $w$ appear qualitatively as the inverse of the hot spot pattern. 

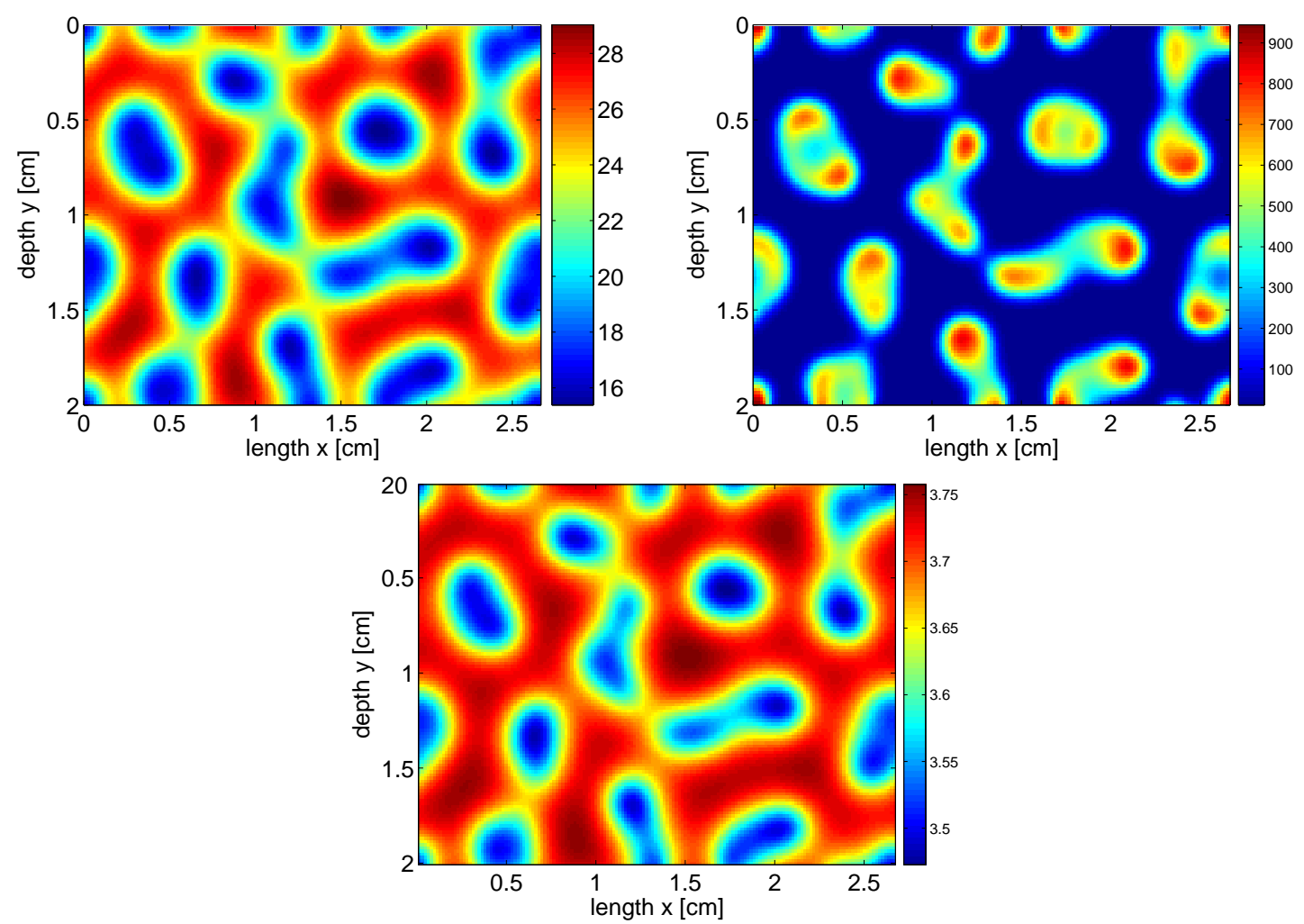

Figure 7: Stationary state of the 2D-simulation of the population density of species $u$ (top left), $v$ (top right) and $w$ (bottom); the population density is illustrated with colors (see colorbar for declaration [dimensionless units]); the initial state is set on equilibrium $S E_{2}$ with random perturbations; the simulation is based on the default parametrization (see Table 2). In this case, $v$ as the activator exhibits a spatial distribution with isolated zones of high concentration of microorganisms ('hot spots'), whereas the distributions of $u$ and $w$ look like the photographic negatives of $v$ ('cold spots'). Note the very different scales for $v$ and $w$.

Thus, the structure evolving in the distribution of $w$ can be referred to as 'cold spot' (or 'gap' [30]) pattern.

In Figure 8, the stationary patterns are obtained by starting with the initial distribution on the randomly perturbed equilibrium $S E_{3}$ (see Eqs. (4.26)); here species $w$ is dominant with high abundance within the spots and it acts as the activator.

If both species $v$ and $w$ would have equal parameters for their growth rates one would expect that the patterns are exactly inverse to each other. We note, that the differences in the shape of the spots are essentially due to the assumption that the two bacteria species are not equal in other parameters.

We have shown that spatially inhomogeneous distributions of substrate and microorganisms can evolve spontaneously, if homogeneous distributions are Turing-unstable. The emergence of patterns is only due to the interaction of nonlinear growth of bacteria and 

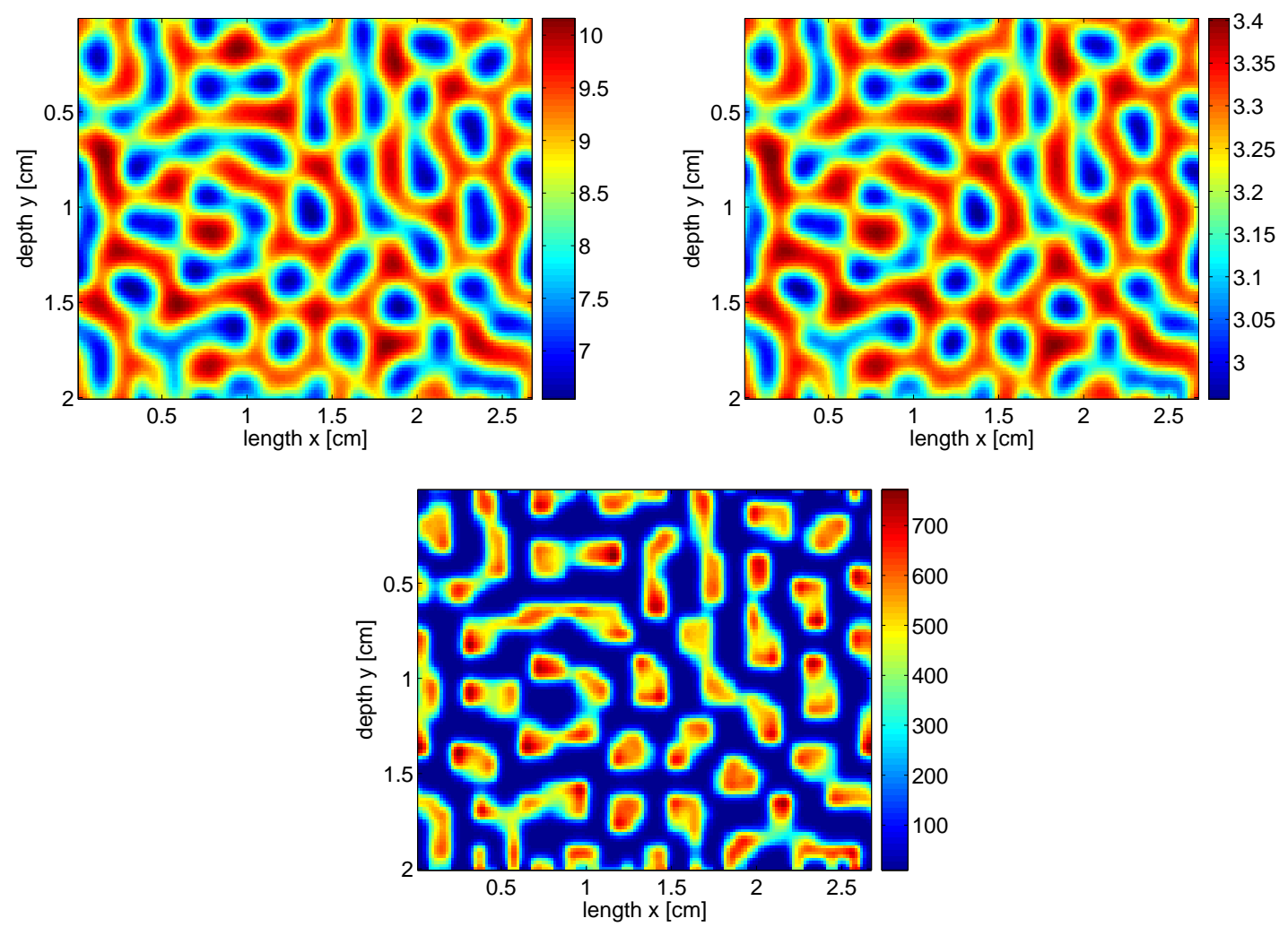

Figure 8: Stationary state of the 2D-simulation of species $u$ (top left), $v$ (top right) and $w$ (bottom); the population density is illustrated with colors (see colorbar for declaration [dimensionless units]); The initial state is set on equilibrium $S E_{3}$ ( $w$ is the activator) with random perturbations; all parameters are taken from Table 2; the stationary patterns are regarded as a transition between spots and stripes.

diffusion, no external gradients are involved. On the average, all patterns we observe for our default parametrization exhibit a diameter of approximately 0.1 to $0.4 \mathrm{~cm}$. Comparing these sizes with the experimentally observed bacterial patterns reported by Thar and Kühl [47], our patterns are about 2 to 4 times bigger. Thar and Kühl [47] have observed that microaerophilic bacteria form mucus veils on top of sulfidic marine sediment exhibiting regular space bacterial patterns ('honeycombs' and their inverse). We therefore conclude that our model exhibits spatial patterns with sizes of a realistic order of magnitude.

\section{$5.2 \quad$ Inhomogeneous supply of electron acceptors}

So far, we have considered electron acceptor concentrations that are constant in space. However, within the sediment the electron acceptor concentrations are depth dependent (see for example the vertical profiles in [22], [54], [53]). Subsequently, these functions also vary with the depth of the sediment. In order to adapt our model to realistic conditions, we 
now take into consideration that the supply of the electron acceptors changes with depth. For this purpose, we do not treat $\rho_{v}$ and $\rho_{w}$ as constants in this section, but as depth dependent functions. For the sake of simplicity, we choose a linear approach. To avoid discontinuities at the top and the bottom of the model's domain, we dismiss the periodic boundary conditions, but apply no-flux conditions instead. At the lateral boundaries we retain the periodic conditions.

The depth dependency of the respiration term acts like an external forcing which we expect to yield to some kind of spatial pattern.

Figure 9 shows the results of the simulation for the default parameter set (see Tab. 2) and $\rho_{v}$ increasing linearly from 0.1 to 0.15 . From Fig. 3, the dependence of the species $v$ and $w$ on respiration is apparent. The range in which $\rho_{v}$ is varied (i.e. [0.1 0.15]) is shown as shaded area. Within this variation we cross critical parameter values (e.g. $\rho_{v}=0.128$ ), where saddle-node bifurcations occur. One particular question we wish to answer with the following simulations is: how is the spatial pattern formation influenced by a local saddlenode bifurcation?

In Figures 9 and 10, the long-term spatial distributions of both bacterial populations are illustrated for the two spatially homogeneous initial distributions $S E_{3}$ and $S E_{2}$, respectively.
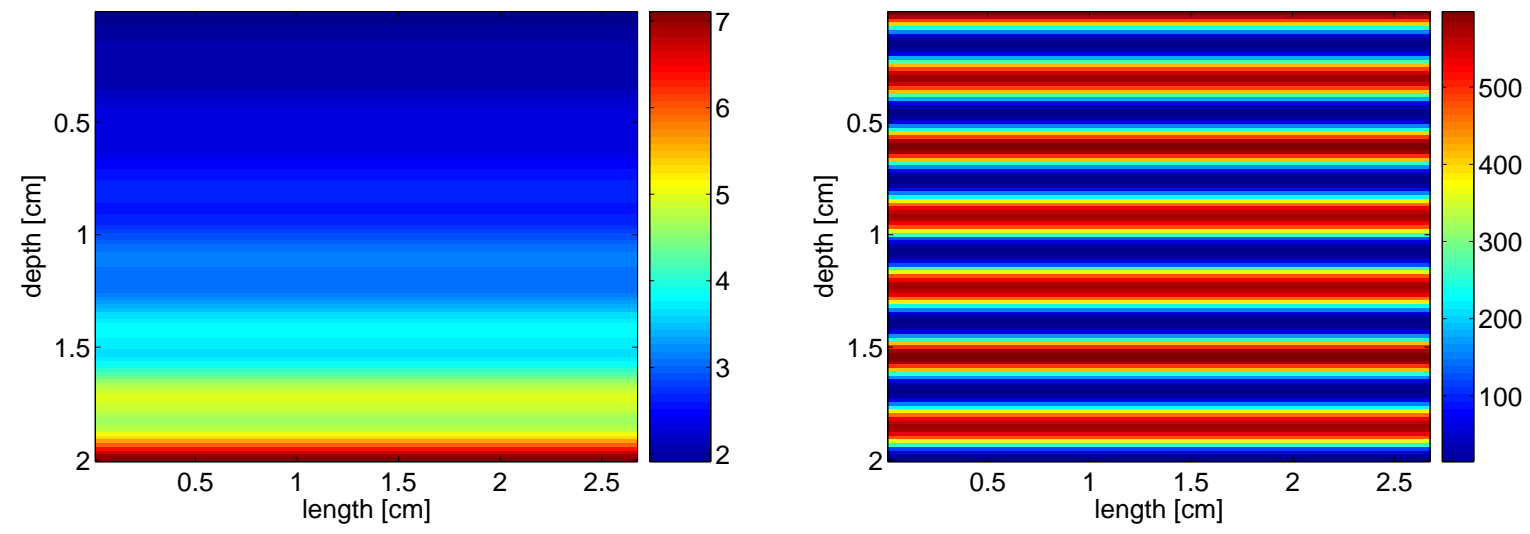

Figure 9: Stationary state of the 2D-simulation of the concentrations (colorbar [dimensionless units]) of the bacterial species $v$ (left) and $w$ (right); the initial state is set on equilibrium $S E_{3}$ ( $w$ is the activator). Parameter $\rho_{v}$ increases linearly from 0.1 to 0.15 (top to bottom); all other parameters are taken from Table 2 .

Let us first concentrate on the equilibrium $S E_{3}$ as the initial distribution. It exhibits a high concentration of the activating species $w$ (see Fig. 3). In this case, the system does not undergo a bifurcation within this range of $\rho_{v}$ (marked in grey). Therefore, we do not expect abrupt transitions in the spatial distributions of the species. Indeed, we clearly see the spatial gradient in the distribution of $v$ (left panel): with increasing depth the concentration of species $v$ increases from approximately 2 to 7 [dimensionless units] almost continuously. This meets our expectations, since $\rho_{v}$ influences species $v$ positively. In Fig. 8 we have observed that the variations in the spatial distribution of the concentration of species $v$ 

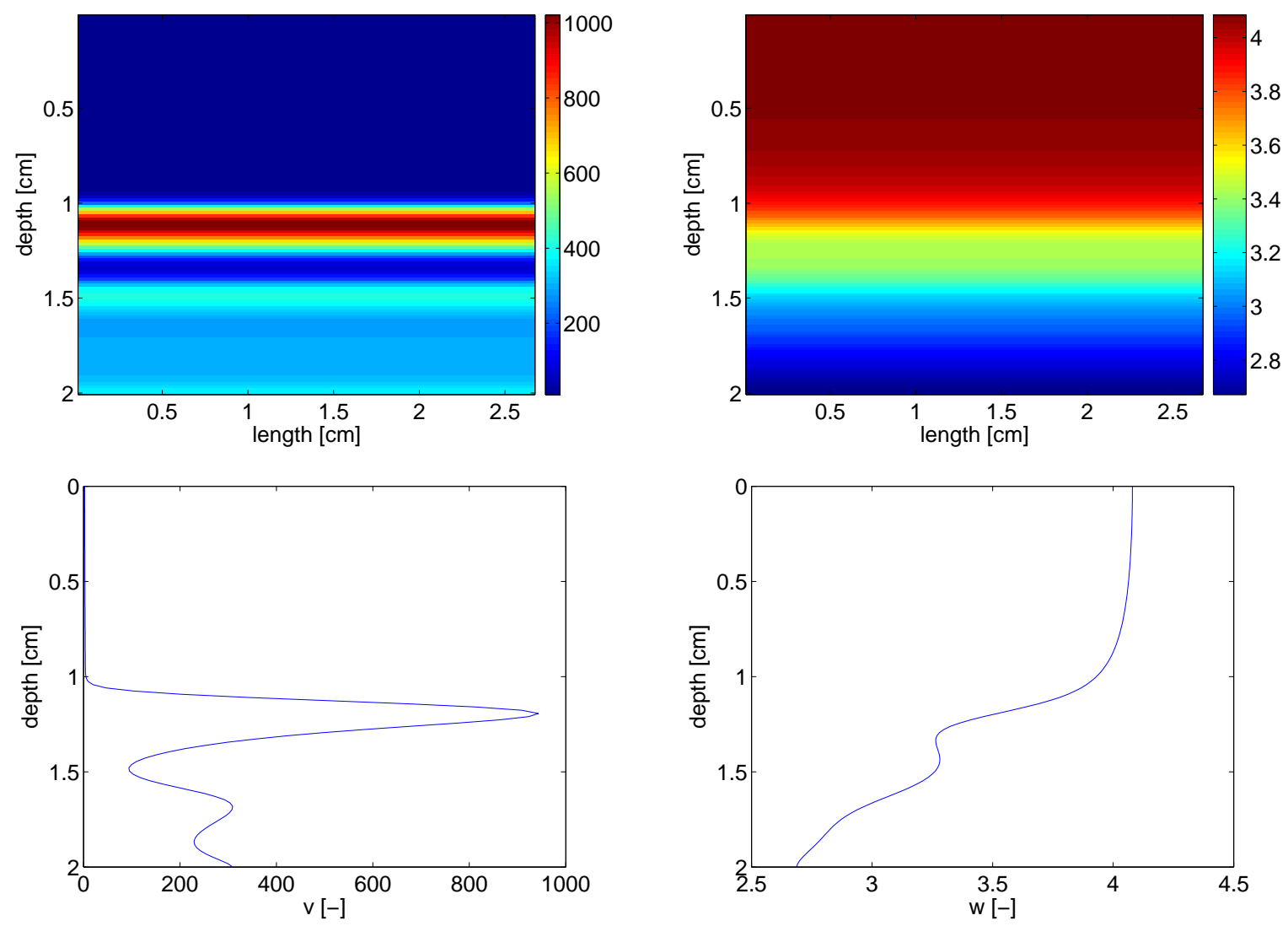

Figure 10: Stationary state of the 2D-simulation of the concentrations (colourbar [dimensionless units]) of the bacterial species $v$ (top left) and $w$ (top right) and vertical sections at length $=0.2 \mathrm{~cm}$ of $v$ (bottom left) and $w$ (bottom right). The initial state is set on equilibrium $S E_{2}$ ( $v$ is the activator). Parameter $\rho_{v}$ increases linearly from 0.1 to 0.15 (top to bottom). All other parameters are taken from Table 2.

are in the range of 3 to 3.4. Structures of this order of magnitude are hardly observable in Figure 9, i.e. the effects of the external forcing superimposes the effects of spontaneous pattern formation.

In the spatial distribution of species $w$, a different situation occurs. Along the spatial gradient, stable band patterns evolve, which exhibit a high concentration of species $w$ (up to a concentration of almost 600 [dimensionless units]). As proved with another simulation (not shown), these banded structures are not imposed by the boundary conditions, but evolve due to the interference between self-organization of the system, i.e. Turing patterns, and the heterogeneous external forcing, i.e. the spatial gradient in $\rho_{v}$. An equivalent observation has been made by [55]. They studied vegetation band patterns on hill slopes using a mathematical model, which applies to drylands. Multiple band patterns coexisting in a wide range of precipitation range were found. In our model the banded structures evolve although species $w$ does not directly depend on $\rho_{v}$. 
In Figure 10, the bacterial populations are initially homogeneously distributed according to the $v$-dominant equilibrium $S E_{2}$. However, due to a saddle-node bifurcation at $\rho_{v} \approx 0.128$ (see Fig. 3) the model cannot converge to a $v$-dominant equilibrium on the entire domain. In the upper half of the domain, in which there is only a weak supply of the electron acceptor $A$, the model will converge to another equilibrium. Indeed, the simulation shows, that a more or less homogeneous distribution corresponding to the $u$-dominant equilibrium $S E_{1}$ evolves. Nevertheless, it is interesting how the system behaves in the lower half of the domain. As you can see in Fig. 10 (left panel) a front evolves, which exhibits a high concentration of species $v$ (up to a concentration of almost 1000 [dimensionless units]) that exceeds the expected value of $\sim 250$, cf. Fig. 3). Below that front, we obtain stripes similar to those observed in Fig. 9 (right panel) but with decreasing amplitudes. Note that the spatial transition from the rather homogeneous upper half to the structured lower one happens rather abrupt. The border between the different regions (the lower one where spatial pattern evolve and the upper one exhibiting a almost homogeneous spatial distribution) is straight and well-defined. In the right panel of Figure 10, the saddle-node bifurcation at a depth of approximately $1.1 \mathrm{~cm}$ is hardly visible, since the resulting transition to the $S E_{1}$ does not cause big difference in the abundance of $w$ (see Figure 3). We clearly see the spatial gradient in the distribution of $w$. In contrast to the previously discussed case, the concentration of species $w$ continuously decreases from 4 to 2.7 [dimensionless units] with increasing depth of the sediment. We expect this behavior, since an increase of $\rho_{v}$ is associated with an enhancement of the living conditions of species $v$. This advantage for species $v$ causes an increase of pressure of competition on species $w$ and thus influences the concentration of species $w$ negatively. The heterogeneous external forcing in form of a spatial gradient again clearly superimposes the self-organized structures.

\section{Conclusions}

The degradation of organic matter in marine sediments is proceeded in a complex network that consists in large parts of microbially catalyzed reactions. Instead of studying the complete network, we focus on two bacteria species that compete for substrate. Both microbial species are using different electron acceptors for respiration. Considering the abundance of bacteria and the substrate as variables, we obtain a three-variable system. We study the dynamics of this system on a $2 D$ spatial vertical domain, taking into account, that both bacteria species and the substrate are subject to diffusion-like motion. Therefore, the entire model can be regarded as a reaction diffusion system, a type of system that has been studied thoroughly in the last decades, in particular with respect to spontaneous pattern formation. The main focus of our paper lies on the investigation of the interaction of diffusion induced pattern formation and inhomogeneous forcing, due to the supply of electron acceptors in the sediments changing with increasing depth.

We get elementary insights into the behaviour of our model by considering its local form (i.e. neglecting transport processes and any spatial dependency of parameters): We show that the model possesses three stationary states that are dominated by either one of the 
microbial species or the substrate. Furthermore, in a broad range of parameters (or, in biological terms, for very variable environmental conditions) we obtain the coexistence of two or even three stable equilibria. In these cases, it depends on the initial concentrations or population densities respectively, what species will be the dominant one in the long-term limit.

Incorporating diffusive transport into the model, we obtain more complicated dynamics. In particular, pattern formation due to Turing instabilities can be observed. We outline the conditions under which the evolution of patterns can occur. In general, it depends on the effect of self-activation: One or eventually both microbial species can possibly excrete signal molecules to stimulate the growth of dormant cells. In this case, pattern formation may occur if the substrate is more mobile than the microbial species. Detailed examination reveals, that the difference in mobility has to be rather large.

In fact, we have determined the Turing space and its boundaries (the critical ratio of diffusion coefficients $\delta_{v, \text { crit }}$ and $\delta_{w, \text { crit }}$ ) analytically as well as numerically, for a general 3component RDS and our specific model [42]. We found that the Turing space crucially depends on the diffusion coefficient of the activating species, whereas the diffusion velocity of the non-autocatalytical species is almost irrelevant for Turing patterns to evolve. Explicit calculation of the critical ratio of diffusion coefficients for the two spatially unstable equilibria $S E_{2}$ and $S E_{3}$ (see Eqs. (4.25) and (4.26), respectively) yields the following values:

$$
\begin{array}{lll}
S E_{2}: & \delta_{w} \in[0, \infty]: & \delta_{v, \text { crit }}=1.9 \times 10^{-4} \\
S E_{3}: & \delta_{v} \in[0, \infty]: & \delta_{w, \text { crit }}=5.1 \times 10^{-5}
\end{array}
$$

However, since it is known that bacteria tend to stick in biofilms or to the sediment matrix, we can consider them as almost immobile. Therefore, the preconditions of pattern formation can be assumed to be fulfilled in many natural systems and particularly in marine sediments. We observe that the patterns, that evolve spontaneously without external gradients, are either stripe structures or spot patterns with either high or low concentrations of bacteria in the isolated zones depending on the parameters. This property seems to be intrinsic for RDS, since it has been reported for models from very different disciplines of science. Furthermore, the same sequence of patterns has been found in [47] in experimental work on pattern formation of marine bacteria. This experimental study gives evidence, that pattern formation is a relevant phenomenon for the degradation processes in marine sediments. Comparing our model results with the experimentally obtained patterns, we find that the size of the spots is in a realistic order of magnitude.

Besides these specific findings for our model, our theoretical analysis allows us to draw a rather general conclusion: In all RDS employing n-dimensional food chains as 'reaction'part, diffusion induced pattern formation presumes the autocatalytic growth of at least one species. This statement is also true for food webs without direct interaction between species of one trophic level.

In the specific model considered in this paper, we meet as a special property of sediments, that the conditions change very rapidly with increasing depth. Hence a non-homogeneous forcing is imposed on the system. An important aspect of this paper is to study how this 
forcing influences pattern formation and whether it can prevent the emergence of patterns, in particular. We find that even if the forcing is not very strong, it exerts massive influence on the patterns that evolve. In particular, we show that a vertical inhomogeneous forcing leads to suppression of horizontal Turing structures and gives rise to the evolution of horizontal bands.

Experimentally, a horizontal banding pattern can frequently be observed when sediment cores are recovered. In the upper anoxic zone of tidal-flat sediments for example, different shades of grey indicate the presence of red-ox interphases caused by bacterial activities occurring in these layers. However, besides the horizontal distribution of bacteria along these boundaries, darker spherical inclusions point towards spatially distributed microbial hotspots.

If the forcing of the model causes the appearance of a saddle-node bifurcation at some depth, another behavior can be observed. At this depth the domain is expected to split up into two parts in which two different equilibria emerge. This division can be related to the formation of a front known from bistable media. In fact though, the situation is more complicated. On the one hand, diffusion may yield a shift of the front towards the region of multistability. On the other hand, we observe that the Turing mechanism leads to the formation of a pulse, that exhibits very high values of the autocatalytic species in the neighborhood of the front.

We also examine the possibility that pattern formation of a dominant species could lead to the evolution of niches in which the counterpart species can invade and become the dominant organism itself. In order to test this hypothesis, we perform simulations in which the concentration of both electron acceptors vary with depth. Regarding the general situation in natural sediments, we have decided to incorporate linear variations with opposite gradients. Unfortunately, our simulations showed no form of invasion. This may be due to the fact that the coexisting equilibria in our model are very different, so that it is difficult to transform one into another. Furthermore the fronts, which we have observed when forcing comes along with the crossing of a saddle-node bifurcation, protect the habitat of one species against the invasion of the other.

We suppose that it is not possible to obtain invasion into patterns, when considering this model with a biological meaningful parametrization. Nevertheless, we claim that this phenomenon is an interesting one that may occur in RDS. Further research is necessary to discuss the possible correlation between pattern formation and invasion.

Acknowledgements. We thank Jan Holstein for beneficial and instructive discussions about the biogeochemical background of our model.

\section{References}

[1] D. Alonso, F. Bartumeus, J. Catalan. Mutual Interference between predators can give rise to Turing spatial patterns. Ecology 83 (2002), 28-34. 
[2] Y.I. Balkarei, A.V. Grigor'Yants, Y.A. Rzhanov, M.I. Elinson. Regenerative Oscillations, spatial-temporal single pulses and static inhomogeneous structures in optically bistable semiconductors. Optics Communications 66 (1988), 161-166.

[3] J.W. Barton, R.M. Ford. Determination of Effective Transport Coefficients for Bacterial Migration in Sand Columns. Applied and Environmental Microbiology 61(9) (1995), 3329-3335.

[4] F. Bartumeus, D. Alonso, J. Catalan. Self-organized spatial structures in a ratiodependent pradeator-prey model. Physica A 295 (2001), 53-57.

[5] M. Baurmann, U. Feudel. Turing Patterns in a Simple Model of a NutrientMicroorganism System in the Sediment. Ecological Complexity 1 (2004), 77-91.

[6] M. Baurmann, W. Ebenhöh, U. Feudel. Turing instabilities and pattern formation in a benthic nutrient-microorganism system. Mathematical Bioscience and Engineering 1 (2004), 111-130.

[7] G. Billen, P. Servais, S. Becquevort. Dynamics of bacterioplankton in oligotrophic and eutrophic aquatic environments: bottom-up or top-down control? Hydrobiologia 207 (1990), 37-42.

[8] M. Billerbeck. Pore water transport and mircrobial activity in intertidal Wadden Sea sediments. PhD Thesis. Universität Bremen, Bremen, 2005.

[9] K. Bosselmann, M.E. Böttcher, M. Billerbeck, E. Walpersdorf, A. Theune, D. de Beer, M. Hüttel, H.-J. Brumsack, B.B. Jørgensen. Iron-sulfur-mangaanese dynamics in intertidal surface sediments in the North Sea. In: BioGeoChemistry of Tidal Flats (Rullkötter, J., ed.). Forschungszentrum Terramare, Wilhelmshaven, 2003, 12.

[10] B.P. Boudreau. Diagenetic models and their implementation: modelling transport and reaction in aquatic sediments. Springer, Berlin, 1997.

[11] A. Bruns, H. Cypionka, J. Overmann, J. Cyclic AMP and Acyl Homoserine Lactones Increase the Cultivatioon Efficiency of Heterotrophic Bacteria from the Central Baltic Sea. Applied and Environmental Microbiology 68(8) (2002), 3978-3987.

[12] A. Bruns, U. Nübel, H. Cypionka, J. Overmann, J. Effect of Signal Compounds and Incubation Conditions on the Culturability of Freshwater Bacterioplankton. Applied and Environmental Microbiology 69(4) (2003), 1980-1989.

[13] S. Camazine, J.-L. Deneubourg, N.R. Franks, J. Sneyd, G. Theraulaz, E. Bonabeau. Self-organisation in biological systems. Princeton University Press, Pinceton, 2001.

[14] V. Castets, E. Dulos, J. Boissonade, P. De Kepper. Experimental evidence of a sustained standing Turing-type nonequilibrium chemical pattern. Physical Review Letters 64 (1990), 2953-2956. 
[15] H. Cypionka. Grundlagen der Mikrobiologie. Springer, Berlin, 2002.

[16] P.N. Froelich, G.P. Klinkhammer, M.L. Bender, N.A. Luedtke, G.R. Heath, D. Cullen, P. Dauphin, D. Hammond, B. Hartman, V. Maynard. Early oxidation of organic matter in pelagic sediments of the eastern equatorial Atlantic: Suboxic diagenesis. Geochim. Cosmochim. Acta 43 (1979), 1075-1090.

[17] P. Glendinning. Stabiliy, instability and chaos: an introduction to the theory of nonlinear differential equations. Cambridge University Press, Cambridge, 1994.

[18] R.R. Haese. Macrobenthic activity and its effects on biogeochemical reactions and fluxes. In: Ocean margin systems (G. Wefer, et al., eds.). Springer, Berlin, 2002, pp. 219-234.

[19] J. von Hardenberg, E. Meron, M. Shachak, Y. Zarmi. Diversity of Vegetation Patterns and Desertification. Physical Review Letters 87(19) (2001), 198101-1(4).

[20] M. Huettel, H. Røy, E. Precht, S. Ehrenhauss, S. Hydrodynamical impact on biogeochemical processes in aquatic sediments. Hydrobiologia 494 (2003), 231-236.

[21] J.M. Holstein. Private communication.

[22] B.B. Jørgensen. Bacteria and Marine Biogeochemistry. In: Marine Geochemistry (H.D. Schulz, M. Zabel, eds.). Springer, Berlin, 2006, pp. 169-206.

[23] C. Klausmeier. Regular and irregular patterns in semiarid vegetation. Science 284 (1999), 1826-1828.

[24] Y.A. Kuznetsov, J.S. Levitin. CONTENT: a multiplatform environment for continuation and bifurcation analysis of dynamical systems. Technical Report. Centrum voor Wiskunde en Informatica, Amsterdam, 1997.

[25] S. Madani, F.J.R. Meysman, J.J. Middelburg. Biogeochemical modeling of sediments from the Santa Barbara Basin (California). In: BioGeoChemistry of Tidal Flats (J. Rullkötter, ed.). Forschungszentrum Terramare, Wilhelmshaven, 2003, pp. 91-93.

[26] P.K. Maini, K.J. Painter, H. Nguyen Phong Chau. Spatial pattern formation in chemical and biological systems. Journal of the Chemical Society, Faraday Trans. 93(20) (1997), 3601-3610.

[27] H. Malchow. Räumliche und raumzeitliche Strukturbildung in Reaktionssystemen mit Diffusion und Advektion. In: Dynamik, Evolution, Strukturen (J.A. Freund, ed.). Verlag Dr. Köster, Berlin, 1996, pp. 229-236.

[28] M. Maruyama. Second Cybernetics - deviation-amplifying mutual causal processes. American scientist 51 (1963), No. 2. 
[29] A.B. Medvinsky, S.V. Petrovskii, I.A. Tikhonova, H. Malchow, B.-L. Li. Spatio-temporal complexity of plankton and fish dynamics in simple model ecosystems. SIAM Review 44(3) (2002), 311-370.

[30] E. Meron, E. Gilad, J. von Hardenberg, M. Shachak, Y. Zarmi. Vegetation patterns along a rainfall gradient. Chaos, Solitons and Fractals 19 (2004), 367-376.

[31] M. Mimura, J.D. Murray. On a Diffusive Prey-Predator Model which exhibits patchiness. Journal of Theoretical Biology 75 (1978), 249-262.

[32] J.D. Murray. Mathematical biology II: Spatial models and biomedical applications. Springer, Berlin, 2003.

[33] G. Nicolis, I. Prigogine. Self-organisation in nonequilibrium systems: from dissipative structures to order through fluctuations. Wiley, Chichester, 1977.

[34] T. Nozakura, S. Ikeuchi. Formation of dissipative structures in galaxies. Astrophysics Journal 279 (1984), 40-52.

[35] M.S. Olson, R.M. Ford, J.A. Smith, E.J. Fernandez. Quanification of Bacterial Chemotaxis in Porous Media Using Magnetic Resonance Imaging. Environmental Science and Technology 38(15) (2004), 3864-3870.

[36] M.S. Olson, R.M. Ford, J.A. Smith, E.J. Fernandez. Mathematical Modeling of Chemotactic Bacterial Transport through a Two-dimensional Heterogeneous Porous Medium. Bioremediation Journal 10 (2006), 13-23.

[37] B. Peña, C. Pérez-García. Stability of Turing patterns in the Brusselator model. Physical Review E 64 (2001), 056213.

[38] D.B. Roszak, R.R. Colwell. Survival Strategies of Bacteria in the Natural Environment. Microbiological Reviews 51 (1987), 365-379.

[39] H. Qian, J.D. Murray. A simple method of parameter space determination for diffusiondriven instability with three species. Applied Mathematics Letters 14 (2001), 405-411.

[40] A.B. Rovinsky. Turing bifurcation and stationary patterns in the Ferroin-catalysed Belousov-Zhabotinsky reaction. Journal of Physical Chemistry 91(17) (1987), 4606-4613.

[41] R.A. Satnoianu, M. Menzinger, P.K. Maini. Turing instabilities in general systems. Journal of Mathematical Biology 41 (2000), 493-512.

[42] Y. Schmitz. Pattern formation of competing microorganisms in sediments: a conceptual model. Diploma thesis. Carl von Ossietzky University, Oldenburg, 2007.

[43] H.D. Schulz. Quantification of Early Diagenesis: Dissolved Constituentes in Pore Water and Signals in the Solid Phase. In: Marine Geochemistry (Schulz, H.D., Zabel, M., eds.). Springer, Berlin, 2006, pp. 75-124. 
[44] L.A. Segel, J.L. Jackson. Dissipative structure: An Explanation and an Ecological Example. Journal of Theoretical Biology 37 (1972), 545-559.

[45] L.H. Stevenson. A Case for Bacterial Dormancy in Aquatic Systems. Microbial Ecology 4 (1978), 127-133.

[46] D. Stiefs, T. Gross, R. Steuer, U. Feudel. Computation and Visualisation of Bifurcation Surfaces. International Journal of Bifurcation and Chaos, in press.

[47] R. Thar, M. Kühl, M. Complex pattern formation of a marine gradient bacteria explained by a simple computer model. FEMS Microbiology Letters 246 (2005), 75-79.

[48] H.R. Thieme. Mathematics in Population Biology. Princeton University Press, Princeton, 2003.

[49] A.M. Turing. The chemical basis of morphogenesis. Philosophical transactions of the Royal Society of London B 237 (1952), 37-72.

[50] P. Van Cappellen, J.-F. Gaillard. Biogeochemical Dynamics in Aquatic Sediments. In: Reactive transport in porous media (P.C. Lichter, C.I. Steefel, E.H. Oelkers, eds.). Reviews in Mineralogy, Vol. 34. Mineralogical Society of America, 1996, pp. 335-376.

[51] D. Walgraef, N.M. Ghoniem. Spatial instabilities and dislocation-loop ordering in irradiated materials. Physical Review B 39 (1989), 8867-8872.

[52] D.B. White. The planforms and onste of convection with a temperature-dependent viscosity. Journal of Fluid Mechanics 191 (1988), 247-286.

[53] R. Wilms, H. Sass, B. Köpke, J. Köster, H. Cypionka, B. Engelen. Specific Bacterial, Archaeal, and Eukaryotic Communities in Tidal-Flat Sediments along a Vertical Profile of Several Meters. Applied and Environmental Microbiology 72(4) (2006), 2756-2764.

[54] R. Wilms, H. Sass, B. Köpke, H. Cypionka, B. Engelen. Methane and sulfate profiles within the subsurface of tidal flats are reflected by the distribution of sulfat-reducing bacteria and methanogenic archaea. FEMS Microbiology Ecology 59(3) (2007), 611-621.

[55] H. Yizhaq, E. Gilad, E. Meron. Banded vegetation: biological productivity and resilience. Physica A 356 (2005), 139-144. 